\title{
Generalizing Ribbons and the Twist of the Lattice Ribbon
}

\author{
Eduardo Dagrosa and Aleksander L Owczarek
}

the date of receipt and acceptance should be inserted later

\begin{abstract}
For a standard or canonical ribbon from differential geometry the topological White's theorem connects the linking number, writhe and total twist of the ribbon. Here we provide an integral expression, analog to the total twist of a canonical ribbon, that connects linking number and writhe of two curves that do not necessarily form a canonical ribbon. First, we apply this integral expression to derive an expression for the writhe of a polygonal curve. Second, but importantly, we revisit the lattice ribbon. Lattice ribbons were introduced some time ago to enable simulation of physical systems modeled by double stranded polymers. Application of the integral expression yields an algorithm for determining the twist of the lattice ribbon. An interesting relation between writhe of the center line of a lattice ribbon and its linking number follows.
\end{abstract}

Keywords Lattice ribbon · White's theorem · Ribbon ·

\section{E. Dagrosa}

Department of Mathematics and Statistics, The University of Melbourne, Victoria 3010

E-mail: e.dagrosa@student.unimelb.edu.au

\section{A. Owczarek}

Department of Mathematics and Statistics, The University of Melbourne, Victoria 3010

E-mail: a.owczarek@ms.unimelb.edu.au 


\section{Introduction}

1.1 Background on canonical ribbons

A canonical ribbon $[3,4,5], \mathscr{C}$, is defined as the doublet $(R, V)$, where $\mathrm{R}$ is a closed curve and $V$ is a constant length vector field on $\mathrm{R}$, that is orthogonal to $\mathrm{R}$. Let $\mathbf{r}(s)\left(\mathbf{r}:[0,2 \pi] \rightarrow \mathbb{R}^{3}\right)$ be a parametrization of $\mathrm{R}$ and $\mathbf{v}(t)$ of $\mathrm{V}$, then

$$
\begin{aligned}
|\mathbf{v}(t)| & =\text { const } \\
\mathbf{v}(t) \cdot \mathbf{r}^{\prime}(t) & =0
\end{aligned}
$$

In addition, it is required that the area traced out by $\mathbf{v}(t)$ does not cross itself. Besides $\mathrm{R}$, the parametrization induces a second boundary curve $\mathrm{B}$ to the ribbon. It has a parametrization $\mathbf{b}(t)=\mathbf{r}(t)+\mathbf{v}(t)$. A theorem accredited to Călugăreanu, Fuller and White $[16,3,4,5,8,14]$ relates the linking number Lk of the boundary curves $\mathrm{R}$ and $\mathrm{B}$ to writhe $\mathrm{Wr}$ and total twist Tw of the ribbon

$$
L k(B, R)=W r(R)+T w^{\mathscr{C}}(B, R) .
$$

where the superscript $\mathscr{C}$ indicates that $R$ and $B$ form a canonical ribbon.

Regardless of whether they form a ribbon, for any two non-intersecting, closed curves $\mathrm{R}$ and $\mathrm{B}$ the linking number is an integer measuring how much the curves intertwine. An informative review on the linking number can found in [15]. To define the linking number, denote the torus by $\mathscr{T}=[0 ; 2 \pi] \times[0 ; 2 \pi]$ and for $\mathbf{x} \in \mathscr{T}$ write $\mathbf{x}=\left(x_{1}, x_{2}\right)$. The Gauss map $\mathbf{G}_{B R}: \mathscr{T} \rightarrow B \times R \rightarrow S^{2}$ of B and $\mathrm{R}$, from the torus onto the two sphere can be defined as

$$
\mathbf{G}_{B R}(\mathbf{x})=\frac{\mathbf{b}\left(x_{2}\right)-\mathbf{r}\left(x_{1}\right)}{\left\|\mathbf{b}\left(x_{2}\right)-\mathbf{r}\left(x_{1}\right)\right\|}
$$

In analogy, a Gauss map corresponding to $R \times R$ is denoted $\mathbf{G}_{R R}(\mathbf{x})$

$$
\mathbf{G}_{R R}(\mathbf{x})=\frac{\mathbf{r}\left(x_{2}\right)-\mathbf{r}\left(x_{1}\right)}{\left\|\mathbf{r}\left(x_{2}\right)-\mathbf{r}\left(x_{1}\right)\right\|}
$$

With the triple product $[\mathbf{a}, \mathbf{b}, \mathbf{c}]=\mathbf{a} \cdot \mathbf{b} \times \mathbf{c}$ and the partial derivative $\partial_{i} \equiv \frac{\partial}{\partial x_{i}}$, the linking number between the two curves $\mathrm{R}$ and $\mathrm{B}$, which is a topological invariant, reads

$$
L k(B, R)=\frac{1}{4 \pi} \int_{\mathscr{T}} d^{2} x\left[\partial_{1} \mathbf{G}_{B R}(\mathbf{x}), \partial_{2} \mathbf{G}_{B R}(\mathbf{x}), \mathbf{G}_{B R}(\mathbf{x})\right]
$$


and hence

$$
L k(B, R)=L k(R, B) .
$$

Since this definition is true for any two curves this symmetry holds true for the generalized ribbon we shall define below. The writhe is a quantity defined on a single closed curve $\mathrm{R}$ and measures its coiling. The writhe of the curve $\mathrm{R}$ reads

$$
W r(R)=\frac{1}{4 \pi} \int_{\mathscr{T} / D} d^{2} x\left[\partial_{1} \mathbf{G}_{R R}(\mathbf{x}), \partial_{2} \mathbf{G}_{R R}(\mathbf{x}), \mathbf{G}_{R R}(\mathbf{x})\right]
$$

where the diagonal $D$ of $\mathscr{T}$ is excluded in the writhe integral.

The third quantity in the CFW theorem (2) is the total twist which can be calculated for a canonical ribbon as

$$
T w^{\mathscr{C}}(B, R)=\frac{1}{2 \pi} \int_{0}^{2 \pi} d t\left[\hat{\mathbf{v}}^{\prime}(t), \hat{\mathbf{r}}^{\prime}(t), \hat{\mathbf{v}}(t)\right]
$$

For a canonical ribbon total twist and writhe are characteristic of the ribbon itself and refer not just to a boundary curve as equations (7, 8 ) might suggest. One may easily convince oneself of this by writing down the twist formula (8) with respect to the curve B. This defines the quantity $T w^{\mathscr{C}}(R, B)$ which is obtained when $\mathbf{r}(t)$ and $\mathbf{v}(t)$ are exchanged for $\mathbf{b}(t)$ and $-\mathbf{v}(t)$ in the total twist formula (8). Importantly, the restrictions of equation (1) of the canonical ribbon guarantee that the twist is symmetric in the boundary curves with

$$
T w^{\mathscr{C}}(B, R)=T w^{\mathscr{C}}(R, B)
$$

so that by equation (2) the writhe of the two boundary curves of a canonical ribbon are equal:

$$
W r(R)=W r(B) \text {. }
$$

In fact this result applies for every curve parametrized $\mathbf{b}_{u}(t)=\mathbf{r}(t)+u \mathbf{v}(t)$ with $u \in[0,1]$ and in particular for the center curve $\mathrm{C}$ of the ribbon which is parametrized when $u=1 / 2$. Therefore, $\operatorname{Wr}(C)=W r(R)=W r(B)$. 
1.2 Results

Our main results are now summarized. In section 2 the concept of a ribbon is generalized, so that certain pairs of closed space curves R and B can be said to form a generalized ribbon. There exist two quantities $T w^{\mathscr{G}}(B, R)$ and $T w^{\mathscr{G}}(R, B)$, which shall be referred to as total twist with respect to $R$ and total twist with respect to $B$, respectively. These satisfy a weak version of the CFW theorem:

Lemma 1 Let $\mathbf{r}(t)$ be a piecewise $C^{1}$ parametrization of a curve $R$ and $\mathbf{b}(t)$ a piecewise $C^{1}$ parametrization of a curve $B$. Let a vector field $V$ on $R$ be parametrized by $\mathbf{v}(t):=\mathbf{b}(t)-\mathbf{r}(t)$. It is assumed that the area traced out by $V$ does not cross itself, then

$$
\begin{aligned}
& L k(B, R)=W r(R)+T w^{\mathscr{G}}(B, R) \\
& L k(R, B)=W r(B)+T w^{\mathscr{G}}(R, B)
\end{aligned}
$$

where the total twist with respect to $R$ reads

$$
\begin{aligned}
T w^{\mathscr{G}}(B, R)= & \lim _{\varepsilon \rightarrow 0} \frac{1}{4 \pi} \int_{0}^{2 \pi} d s \int_{0}^{\pi} d \theta \\
& \times\|\mathbf{r}(s-\varepsilon \cos \theta)+\varepsilon \sin \theta \mathbf{v}(s-\varepsilon \cos \theta)-\mathbf{r}(s)\|^{-3} \\
& \times[\mathbf{r}(s-\varepsilon \cos \theta)-\mathbf{r}(s)+\varepsilon \sin \theta \mathbf{v}(s-\varepsilon \cos \theta) \\
& \mathbf{r}^{\prime}(s-\varepsilon \cos \theta)-\mathbf{r}^{\prime}(s)+\varepsilon \sin \theta \mathbf{v}^{\prime}(s-\varepsilon \cos \theta) \\
& \varepsilon \sin \theta \mathbf{r}^{\prime}(s-\varepsilon \cos \theta)+\varepsilon \cos \theta \mathbf{v}(s-\varepsilon \cos \theta) \\
& \left.+\varepsilon^{2} \sin ^{2} \theta \mathbf{v}^{\prime}(s-\varepsilon \cos \theta)\right]
\end{aligned}
$$

An analogous formula holds for total twist with respect to $B T w^{\mathscr{G}}(R, B)$.

For our purposes a $C^{1}$ parametrization $\mathbf{r}(t)$ shall be called almost $C^{2}$, when $\mathbf{r}^{\prime \prime}(t)$ is continous almost everywhere. Also, $\mathbf{r}^{\prime \prime}(t)$ may vanish on intervals.

Lemma 2 With the definition as in lemma 1, but with $\mathbf{r}(t)$ being almost $C^{2}$, the total twist with respect to $R$ becomes a one dimensional integral 


$$
T w^{\mathscr{G}}(B, R)=\frac{1}{2 \pi} \int_{0}^{2 \pi} d t \frac{\left|\mathbf{r}^{\prime}\right|}{\left|\mathbf{r}^{\prime}\right|^{2}|\mathbf{v}|^{2}-\left\langle\mathbf{r}^{\prime}, \mathbf{v}\right\rangle^{2}}\left(\left[\mathbf{v}^{\prime}, \mathbf{r}^{\prime}, \mathbf{v}\right]+\frac{\left\langle\mathbf{r}^{\prime}, \mathbf{v}\right\rangle}{\left|\mathbf{r}^{\prime}\right|^{2}}\left[\mathbf{v}, \mathbf{r}^{\prime}, \mathbf{r}^{\prime \prime}\right]\right)
$$

An analogous formula holds for $T w^{\mathscr{G}}(R, B)$ when corresponding conditions for $\mathbf{b}(t)$ are satisfied.

In the total twist formula, equation (13), the dependencies on the parameter $t$ are suppressed, eg $\mathbf{r}^{\prime}=\mathbf{r}^{\prime}(t)$, etc. Note that we can also express the twist formula by setting all $|\bullet|=1$ and normalizing the remaining vector quantities. The proof of the lemmas $(1,2)$ builds on a method used to prove the CFT theorem [7] and the associated total twist, by introducing a generalized Gauss map on an appropriately defined integration space. In contrast to before-mentioned publication [7], the language of abstract differential geometry is not used in this paper. This comes at the expense of having to make a somewhat intuitive ansatz. Regarding the twist formula (13), suppose there exists a parametrization such that $\left\langle\mathbf{r}^{\prime}(s), \mathbf{v}(s)\right\rangle=0$ and $|\mathbf{v}(s)|=$ const, then the second term in equation (13) vanishes and the first term reduces to the twist of the canonical ribbon $T w^{\mathscr{C}}(B, R)$ (equation (8)). Therefore, $T w^{\mathscr{G}}(B, R)$ includes $T w^{\mathscr{C}}(B, R)$ as the special case where $R$ and $B$ form a canonical ribbon. Therefore, let $T w(B, R) \equiv T w^{\mathscr{G}}(B, R)$ so that there is no need to distinguish by superscripts anymore. The twist formula may be regarded as a tool that helps to compute the writhe of a curve $R$ when the linking number with $B$ is known or vice versa compute the linking number when the writhe is known. Note that alternatively, it is always possible to transform $B$, but without changing $L k(B, R)$, until $B$ and $R$ form a canonnical ribbon. In fact, given a vector field $\mathbf{v}(t)$, we may orthogonalize it with respect to $R$ as $\mathbf{v}^{\perp}(t)=\mathbf{v}(t)-<\mathbf{v}(t), \mathbf{r}^{\prime}(t)>\mathbf{r}^{\prime}(t)$. The vectorfield parametrized by $\left.\mathbf{v} \hat{(t}\right)^{\perp}$ forms a canonical ribbon with $R$. Plugging $\mathbf{v} \hat{(t)}{ }^{\perp}$ into the twist formula of the canonical ribbon (8) recovers formula (13). In addition, note that the writhe difference between two curves is given by the local integral

$$
W r(B)-W r(R)=T w(B, R)-T w(R, B)
$$


However, this expression (eq 14) is not as compact as the traditional formula for the writhe difference [9] proved in [2].

In section 3 the results of section 2 (eqs $(13,11))$ are used to derive an expression for the writhe of a polygonal curve $R^{P}$. Such an expression is useful in simulations that require efficient computation of the writhe of a polygonal curve. Before considering a polygonal curve, consider a closed $C^{1}$ curve $R$. Choose a vector $\mathbf{d}$ whose direction does not coincide with the orientation of any of the tangent vectors to the curve $R$. The set formed by all normalized tangent vectors is called the tantrix $T_{R}$ of $R$. Then, there exists an $\varepsilon$ such that $R$ and its pushed off curve $R+\varepsilon \mathbf{d}$ form a generalized ribbon with $\mathbf{v}(t)=\varepsilon \mathbf{d}=$ const. The writhe of $R$ may then be expressed as the difference between linking number and generalized total twist eqs $(13,11)$. The total twist integral contains two triple product. The first triple vanishes due to the occurrence of $\mathbf{v}^{\prime}(t)$. It remains

$$
W r(R)=L k(R+\varepsilon \mathbf{d}, R)-\frac{1}{2 \pi} \int_{0}^{2 \pi} d s \frac{\left|\mathbf{r}^{\prime}(s)\right|^{-1}\left\langle\mathbf{r}^{\prime}(s), \mathbf{d}\right\rangle}{\left|\mathbf{r}^{\prime}(s)\right|^{2}|\mathbf{d}|^{2}-\left\langle\mathbf{r}^{\prime}(s), \mathbf{d}\right\rangle^{2}}\left[\mathbf{d}, \mathbf{r}^{\prime}(s), \mathbf{r}^{\prime \prime}(s)\right]
$$

The linking number between the curve $R$ and its pushed off curve $R+\varepsilon \mathbf{d}$ is called directional writhe $W r_{d}(R)$. Returning to the polygonal curve, equation (15) cannot be applied because the second derivative $\mathbf{r}^{\prime \prime}$ is not defined at vertices. This problem will be circumvented by connecting edges at vertices through parts of a circle of radius $\delta r$. The curve which emerges from $R^{P}$ by applying this procedure is $C^{1}$ and shall be denoted $R_{\delta r}$. The limit $\delta r \rightarrow 0$ under which $R_{\delta r} \rightarrow R^{P}$ shall be called the polygonal limit. The tantrix of $R^{P}$ is defined as $T_{R^{P}}:=T_{R_{\delta r}}$. The following lemma regarding the writhe of a polygonal curve can be derived

Lemma 3 Let $R^{P}$ be an oriented, non-intersecting, closed, polygonal curve of length $N$. Then, $R^{P}$ is formed by line segments whose orientation and length is given by vectors $\mathbf{a}_{i}$. Let $\mathbf{d}$ be any vector so that $\hat{\mathbf{d}} \notin T_{R^{P}}$. Then, the writhe of $R^{P}$ reads

$$
\begin{aligned}
W r\left(R^{P}\right)= & W r_{d}\left(R^{P}\right)-\frac{1}{2 \pi} \sum_{i=1}^{N} \operatorname{sgn}\left(d_{i}^{\perp}\right)\left\{\arctan \left(\frac{d_{i-1}-d_{i} \cos \alpha_{i}}{\left|d_{i}^{\perp}\right| \sin \alpha_{i}}\right)\right. \\
& \left.+\arctan \left(\frac{d_{i}-d_{i-1} \cos \alpha_{i}}{\left|d_{i}^{\perp}\right| \sin \alpha_{i}}\right)\right\}
\end{aligned}
$$


where

$$
\begin{gathered}
\cos \alpha_{i}=\left\langle\hat{\mathbf{a}}_{i-1}, \hat{\mathbf{a}}_{i}\right\rangle \\
d_{i}=\left\langle\mathbf{d}, \hat{\mathbf{a}}_{i}\right\rangle \\
d_{i}^{\perp} \sin \alpha_{i}=\left[\mathbf{d}, \hat{\mathbf{a}}_{i-1}, \hat{\mathbf{a}}_{i}\right]
\end{gathered}
$$

In addition, it is found that the polygonal limit has no effect on the generalized twist

$$
T w\left(R^{P}+\varepsilon \mathbf{d}, R^{P}\right)=T w\left(R_{\delta r}+\varepsilon \mathbf{d}, R_{\delta r}\right)
$$

As a consequence, the writhe difference between a polygonal curve $R^{P}$ and the corresponding $C^{1}$ curve $R_{\delta r}$ must be an integer. In particular $\delta r$ can always be chosen small enough so that

$$
W r\left(R^{P}\right)=W r\left(R_{\delta r}\right)
$$

The same result eq (18) was found in [6].

Traditionally, different but analogous results to equation (16) have been derived $[1,12]$ by constructing a second curve $B$ around a given curve $R$, so that a canonical ribbon $\mathscr{C}$ is formed. Then, the relation

$$
W r(R)+T w(B, R)=W r_{d}(R)+T w_{d}(B, R)
$$

is used where $T w_{d}(B, R)$ is called the directional twist. This quantity is usually defined as the crossing number between $R$ and $B$, so that $R$ and $B$ cross locally in a projection diagram along the direction $\hat{\mathbf{d}}$.

Note that by comparing eq (19) with eq (15) one concludes that for any canonical ribbon on $R$

$$
T w(B, R)-T w_{d}(B, R)=\frac{1}{2 \pi} \int_{0}^{2 \pi} d s \frac{\left|\mathbf{r}^{\prime}(s)\right|^{-1}\left\langle\mathbf{r}^{\prime}(s), \mathbf{d}\right\rangle}{\left|\mathbf{r}^{\prime}(s)\right|^{2}|\mathbf{d}|^{2}-\left\langle\mathbf{r}^{\prime}(s), \mathbf{d}\right\rangle^{2}}\left[\mathbf{d}, \mathbf{r}^{\prime}(s), \mathbf{r}^{\prime \prime}(s)\right]
$$




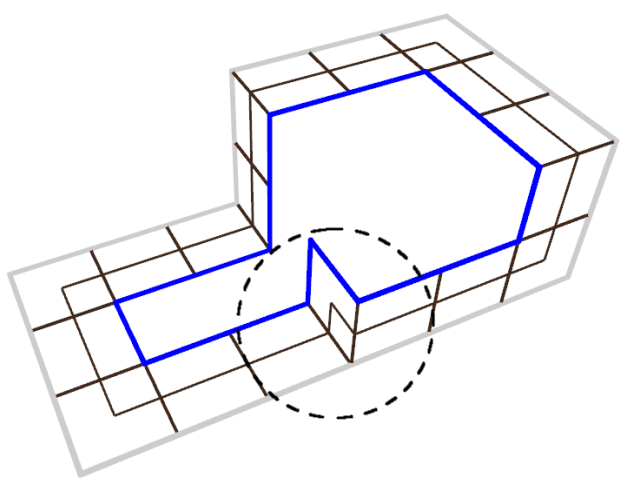

Fig. 1 Example of a lattice ribbon with boundary curves $R$ (light) and $B$ (dark ). The ribbon edges are the lines that connect $R$ and $B$. The curve in the middle is the center curve $C$. The writhes of the boundary curves differ $W r(B)=0, W r(R)=-1 / 4$. Also, $W r(C)=0$. The circled configuration consists of a $B$ corner plaquette between two ordinary plaquettes so that $B$ twists around $R$ by an angle of $\pi / 2$. When the lattice ribbon is build from these configurations and is otherwise planar, the twist can be inferred. For a general lattice ribbon, it is not so trivial to identify the source of total twist.

In section 4 some new results regarding the lattice ribbon are proved. A lattice ribbon [11], $\mathscr{L}$, is constructed by concatenating unit squares (plaquettes) on the simple cubic lattice $\left(Z^{3}\right)$ as shown in figure 1 . Two of the four edges of each plaquette are part of two boundary curves of the ribbon, which shall be denoted by $R$ and $B$. When a plaquette has two edges belonging to the curve $R(B)$, it is called a $R$ (B) corner plaquette. Otherwise, it is called ordinary. When the boundary curves are closed, the lattice ribbon is referred to as closed (and oriented).

The lattice ribbon, $\mathscr{L}$, is not canonical ribbon, because given the two boundary curves of the lattice ribbon, in general it is not possible to find an orthogonal vector field $\mathrm{V}$ that connects every point on $\mathrm{R}$ with a point on $\mathrm{B}$. This can be seen in figure 1 , which also makes clear that in general $W r(R) \neq W r(B)$. Both linking number and writhe are well defined for a lattice ribbon, so that in previous work on the lattice ribbon [10], total twist was defined as $T w(B, R):=L(B, R)-W r(R)$. In a paper [13] on open and directed lattice ribbons, right- and left-handed twisting configurations formed from three plaquettes (as circled in the figure) were identified. However, it was not considered how these configurations would have to be counted for equation (2) to hold. For an arbitrary lattice ribbon, the challenge lies in identifying the 


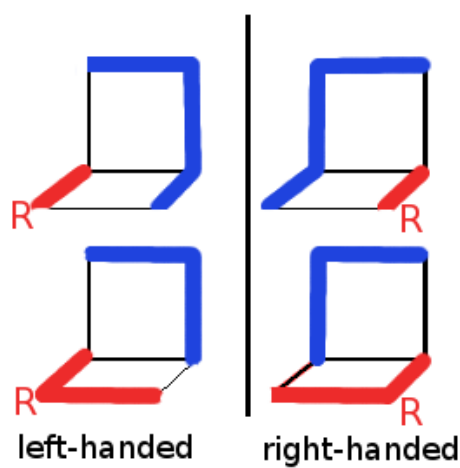

Fig. 2 All local twisting configurations that can contribute to $T w(B, R)$. The left-handed configurations contribute $-1 / 4$, the right-handed $+1 / 4$. A $B$ corner plaquette may not be considered in more than one configuration. The right handed configurations emerge from the left handed by reflection at a plane.

configurations which have to be counted to yield the proper twist $T w(B, R)$. The twist formula will be applied to derive the following lemma

Lemma 4 Let $\mathscr{L}$ be any lattice ribbon with boundary curves $R$ and $B$. Whenever a $B$-corner plaquette has at least one neighboring plaquette with perpendicular orientation, a contribution to $T w(B, R)$ is made. This contribution has value $1 / 4$ when the configuration formed by the two plaquettes is right-handed and $-1 / 4$ when it is left handed.

The relevant configurations are shown in figure 2.

Finally, a lemma regarding the center curve of the lattice ribbon is proved

Lemma 5 For a closed lattice ribbon with boundary curves $R, B$ and center curve $C$

$$
W r(C)=\operatorname{Lk}(R, B)
$$

Note from figure 1 that the center curve $C$ is a self avoiding polygon (SAP) on the simple cubic lattice with half integer spacing $(\mathbb{Z} / 2)^{3}$. This lattice is trivially equivalent to $\mathbb{Z}^{3}$. A general SAP on this lattice is known [10] to have writhe $W r$ so that $4 \mathrm{Wr}$ is an integer. Therefore, according to lemma 5, not all SAP on the half integer lattice can be center curves of a lattice ribbon. This is discussed further in the appendix A. 


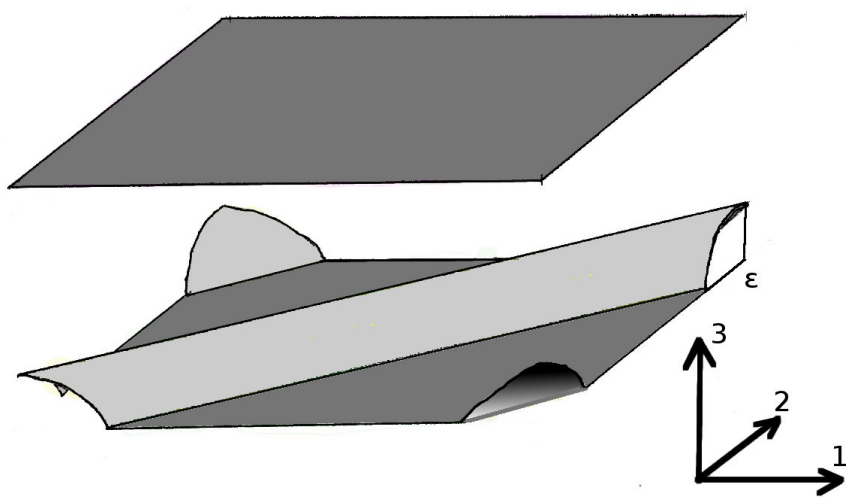

Fig. 3 The top surface $\mathrm{P}$ represents the torus at $x_{3}=1$. The bottom surface $G_{\varepsilon}$ represents the torus at $x_{3}=0$ with an $\varepsilon$ sized strip excluded around the diagonal. The light shaded surface $Y_{\varepsilon}$ is an $\varepsilon$ sized half tube . Note that because of the periodicity of the torus parts of the tube reappear at the other side. The three surfaces enclose the domain $V_{\varepsilon}$.

\section{Generalized Ribbon}

Define a generalized closed ribbon by discarding the restrictions in equations (1)

Definition 1 A generalized closed ribbon $\mathscr{G}$ consists of a closed, piecewise $C^{1}$ curve $\mathrm{R}$ and a piecewise $C^{1}$ vector field $\mathrm{V}$ along $\mathrm{R}$ that does not intersect itself.

With $\mathbf{r}(s)$ a parametrization of $\mathrm{R}, \mathbf{v}(s)$ a parametrization of $\mathrm{V}$ and $u \in[0,1]$ the points of $\mathscr{G}$ are parametrized by $\mathscr{G}(s, u)=\mathbf{r}(s)+u \mathbf{v}(s)$. The condition that $\mathrm{V}$ does not intersect itself is equivalent to $\mathscr{G}(s, u)$ being bijective. A second boundary curve B is defined by $\mathbf{r}(s)+\mathbf{v}(s)$. In the introduction linking number and writhe are expressed as integrals on the product manifold $R \times B$ and $R \times R / D$, respectively. The latter are parametrized from the torus. A geometric explanation for the CFW theorem can be found by extending, the torus by one dimension to form the space $\mathscr{T} \times \mathbb{R}$. Denote a point in this space by the coordinate triplet $\mathbf{x}=\left(x_{1}, x_{2}, x_{3}\right)$. Define a closed subset $V=\mathscr{T} \times[0,1]$ of $\mathscr{T} \times \mathbb{R}$ and remove an epsilon sized tube along the diagonal of the bottom of $\mathrm{V}$ to form the region $V_{\varepsilon}$ depicted in figure 3 . The boundary of the parametrization region $V_{\varepsilon}$ is formed by the top $\mathrm{P}$, bottom $G_{\varepsilon}$ and tube shaped $Y_{\varepsilon}$ surfaces, which themselves can be parametrized as 


$$
\begin{aligned}
P & =\{(s, t, 1) \mid s \in S, t \in S\} \\
G_{\varepsilon} & =\{(s, t, 0):|s-t|>\varepsilon \mid s \in S, t \in S\} \\
Y_{\varepsilon} & =\{(s, s-\varepsilon \cos \theta, \varepsilon \sin \theta) \mid s \in S, 0 \leq \theta \leq \pi\}
\end{aligned}
$$

On $V_{\varepsilon}$ define the generalized Gauss map

$$
\mathbf{g}(\mathbf{x})=\frac{\mathbf{r}\left(x_{2}\right)+x_{3} \mathbf{v}\left(x_{2}\right)-\mathbf{r}\left(x_{1}\right)}{\left\|\mathbf{r}\left(x_{2}\right)+x_{3} \mathbf{v}\left(x_{2}\right)-\mathbf{r}\left(x_{1}\right)\right\|}
$$

and the vector field $\psi: V_{\varepsilon} \rightarrow \mathbb{R}^{3}$

$$
\psi(\mathbf{x})=\frac{1}{4 \pi}\left(\begin{array}{c}
{\left[\mathbf{g}, \partial_{2} \mathbf{g}, \partial_{3} \mathbf{g}\right]} \\
{\left[\partial_{1} \mathbf{g}, \mathbf{g}, \partial_{3} \mathbf{g}\right]} \\
{\left[\partial_{1} \mathbf{g}, \partial_{2} \mathbf{g}, \mathbf{g}\right]}
\end{array}\right)
$$

Regarding $\Psi$, note that when the surface traced out by $\mathbf{v}$ does not cross itself, $\psi(\mathbf{x})$ has no singularities on $V_{\varepsilon}$. Further, considering that $\mathbf{g}$ is a unit vector, all its partial derivatives lie in the plane perpendicular to $\mathbf{g}$ implying $\left[\partial_{1} \mathbf{g}, \partial_{2} \mathbf{g}, \partial_{3} \mathbf{g}\right]=0$. Further, as long as the derivatives of $\mathbf{r}$ and $\mathbf{b}$ are defined everywhere, it holds that $\partial_{i} \partial_{j} \mathbf{g}=\partial_{j} \partial_{i} \mathbf{g}$. Therefore, the divergence of $\psi$ vanishes by construction

$$
\operatorname{div}(\psi)=0
$$

Note that $\mathbf{r}$ and $\mathbf{b}$ being piecewise $C^{1}$ is a sufficient condition for the vanishing of the divergence, which when combined with

Gauss's divergence theorem

$$
\int_{V} d^{3} x \operatorname{div}(\psi)=\int_{\partial V} \psi \cdot d \mathbf{A}
$$

gives

$$
\begin{aligned}
0 & =\int_{\partial V_{\varepsilon}} \psi \cdot d \mathbf{A} \\
& =\int_{P} \psi \cdot d \mathbf{A}-\int_{G_{\varepsilon}} \psi \cdot d \mathbf{A}-\int_{Y_{\varepsilon}} \psi \cdot d \mathbf{A}
\end{aligned}
$$

where the minus signs results when the surface normals of $G$ and $Y$ are oriented with positive $x_{3}$ component. Note that $\mathbf{r}$ and $\mathbf{b}$ being piecewise $C^{1}$ and $\mathrm{V}$ not crossing 
is a sufficient condition to guarantee a well defined integral of the divergence of $\operatorname{div}(\psi)$ and the surface integrals. By comparison of equation (21) with equation (5) one identifies the integral over $\mathrm{P}$ with the linking number of $R$ and $B$. In the limit $\varepsilon \rightarrow 0$, the integral over $\mathrm{G}$ is identified (eq. (7)) as the writhe of $\mathrm{R}$ and the integral over $Y_{\varepsilon}$ shall be called the generalized total twist $T w(B, R)$ with respect to $\mathrm{R}$ (In the following $T w(B, R)$ is often just referred to as twist). Therefore, equation (21) is essentially the CFW theorem and the first part of lemma 1 is proven.

In order to evaluate the twist integral $\int_{Y_{\varepsilon}} \psi(\mathbf{x}) \cdot d \mathbf{A}$, note that with $\mathbf{p}\left(x_{1}, x_{2}, x_{3}\right)=$ $\mathbf{r}\left(x_{2}\right)+x_{3} \mathbf{v}\left(x_{2}\right)-\mathbf{r}\left(x_{1}\right)$

$$
\left[\mathbf{g}, \partial_{i} \mathbf{g}, \partial_{j} \mathbf{g}\right]=\frac{\left[\mathbf{p}, \partial_{i} \mathbf{p}, \partial_{j} \mathbf{p}\right]}{(\mathbf{p} \cdot \mathbf{p})^{\frac{3}{2}}}
$$

In the transformed coordinates on $Y_{\varepsilon}$

$$
\mathbf{x}_{Y_{\varepsilon}}(s, \theta)=\left(\begin{array}{c}
s \\
s-\varepsilon \cos \theta \\
\varepsilon \sin \theta
\end{array}\right)
$$

the integral reads

$$
\int_{Y_{\varepsilon}} \psi(\mathbf{x}) \cdot d \mathbf{A}=\varepsilon \int_{0}^{2 \pi} d s \int_{0}^{\pi} d \theta \psi(s, \theta) \cdot\left(\begin{array}{c}
\cos \theta \\
-\cos \theta \\
\sin \theta
\end{array}\right)
$$

The derivatives transform as

$$
\begin{aligned}
& \partial_{1} \rightarrow \tilde{\partial}_{1}=\partial_{s}-\frac{1}{\varepsilon} \sin \theta \partial_{\theta}+\cos \theta \partial_{\varepsilon} \\
& \partial_{2} \rightarrow \tilde{\partial}_{2}=\frac{1}{\varepsilon} \sin \theta \partial_{\theta}-\cos \theta \partial_{\varepsilon} \\
& \partial_{3} \rightarrow \tilde{\partial}_{3}=\frac{1}{\varepsilon} \cos \theta \partial_{\theta}+\sin \theta \partial_{\varepsilon}
\end{aligned}
$$

so that after some algebraic transformations

$\psi(s, \theta) \cdot\left(\begin{array}{c}\cos \theta \\ -\cos \theta \\ \sin \theta\end{array}\right)=\frac{1}{4 \pi} \frac{\varepsilon^{-1}}{(\mathbf{p} \cdot \mathbf{p})^{\frac{3}{2}}}\left[\mathbf{p}(s, \theta, \varepsilon), \partial_{s} \mathbf{p}(s, \theta, \varepsilon), \partial_{\theta} \mathbf{p}(s, \theta, \varepsilon)\right]$ 
When the explicit form of $\mathbf{p}$ and its derivatives is inserted into the last equation (23), together with the identification of $T w(B, R)$ as the limit $\varepsilon \rightarrow 0$ of equation 22, the proof of lemma 1 is concluded.

In the following it is assumed that $\mathbf{r}(s-\varepsilon \cos \theta), \mathbf{r}^{\prime}(s-\varepsilon \cos \theta), \mathbf{v}(s-\varepsilon \cos \theta)$ and $\mathbf{v}^{\prime}(s-\varepsilon \cos \theta)$ can be approximated by Taylor expansion around $s$. Then, the quantity $\mathbf{p}$ and its derivatives read

$$
\begin{aligned}
\mathbf{p}(s, \theta, \varepsilon) & =\mathbf{r}(s-\varepsilon \cos \theta)-\mathbf{r}(s)+\varepsilon \sin \theta \mathbf{v}(s-\varepsilon \cos \theta) \\
& =\varepsilon\left\{-\cos \theta \mathbf{r}^{\prime}(s)+\sin \theta \mathbf{v}(s)\right\}+O\left(\varepsilon^{2}\right) \\
\partial_{s} \mathbf{p}(s, \theta, \varepsilon) & =\mathbf{r}^{\prime}(s-\varepsilon \cos \theta)-\mathbf{r}^{\prime}(s)+\varepsilon \sin \theta \mathbf{v}^{\prime}(s-\varepsilon \cos \theta) \\
& =-\varepsilon \cos \theta \mathbf{r}^{\prime \prime}(s)+\varepsilon \sin \theta \mathbf{v}^{\prime}(s)+O\left(\varepsilon^{2}\right) \\
\partial_{\theta} \mathbf{p}(s, \theta, \varepsilon) & =\varepsilon \sin \theta \mathbf{r}^{\prime}(s)+\varepsilon \cos \theta \mathbf{v}(s)+O\left(\varepsilon^{2}\right)
\end{aligned}
$$

With the above, relation (23) becomes

$$
\begin{aligned}
\psi(s, \theta) \cdot\left(\begin{array}{c}
\cos \theta \\
-\cos \theta \\
\sin \theta
\end{array}\right)= & \frac{\varepsilon^{-1}}{4 \pi}\left\{\frac{\sin \theta\left[\mathbf{r}^{\prime}, \mathbf{v}, \mathbf{v}^{\prime}\right]+\cos \theta\left[\mathbf{r}^{\prime}, \mathbf{r}^{\prime \prime}, \mathbf{v}\right]}{\left(\cos ^{2} \theta\left|\mathbf{r}^{\prime}\right|^{2}-2 \cos \theta \sin \theta \mathbf{r}^{\prime} \cdot \mathbf{v}+\sin ^{2} \theta|\mathbf{v}|^{2}\right)^{\frac{3}{2}}}\right. \\
& +O(\varepsilon)\}
\end{aligned}
$$

which when used in relation (22) and taking the limit $\varepsilon \rightarrow 0$ gives the total twist

$$
T w(B, R)=\frac{1}{4 \pi} \int_{0}^{2 \pi} d s \int_{0}^{\pi} d \theta \frac{\sin \theta\left[\mathbf{r}^{\prime}, \mathbf{v}, \mathbf{v}^{\prime}\right]+\cos \theta\left[\mathbf{r}^{\prime}, \mathbf{r}^{\prime \prime}, \mathbf{v}\right]}{\left(\cos ^{2} \theta\left|\mathbf{r}^{\prime}\right|^{2}-2 \cos \theta \sin \theta \mathbf{r}^{\prime} \cdot \mathbf{v}+\sin ^{2} \theta|\mathbf{v}|^{2}\right)^{\frac{3}{2}}}
$$

Note, that the dependence on the integration variable $s$ of the vector quantities has been suppressed for formatting reasons. In order to perform the integral over $\theta$, let $t(x)=\cos (x), \sin (x)$, then an integral function of

$$
f_{t}(x)=\frac{t(x)}{\left(a \sin ^{2}(x)+2 b \cos (x) \sin (x)+c \cos ^{2}(x)\right)^{\frac{3}{2}}}
$$


can be found by making the ansatz $F_{t}(x)=\frac{A_{t} \sin (x)+B_{t} \cos (x)}{\sqrt{a \sin ^{2}(x)+2 b \cos (x) \sin (x)+c \cos ^{2}(x)}}$ and determining $A_{t}$ and $B_{t}$ from the derivative $F_{t}^{\prime}(x) \stackrel{!}{=} f_{t}(x)$. This allows to perform the integration over $\theta$ yielding the result of lemma 2

$$
\begin{aligned}
T w(B, R)= & \frac{1}{2 \pi} \int_{0}^{2 \pi} d t \frac{\left|\mathbf{r}(t)^{\prime}\right|}{\left|\mathbf{r}^{\prime}(t)\right|^{2}|\mathbf{v}(t)|^{2}-\left\langle\mathbf{r}^{\prime}(t), \mathbf{v}(t)\right\rangle^{2}} \\
& \times\left\{\left[\mathbf{v}^{\prime}(t), \mathbf{r}^{\prime}(t), \mathbf{v}(t)\right]+\frac{\left\langle\mathbf{r}^{\prime}(t), \mathbf{v}(t)\right\rangle}{\left|\mathbf{r}^{\prime}(t)\right|^{2}}\left[\mathbf{v}(t), \mathbf{r}^{\prime}(t), \mathbf{r}^{\prime \prime}(t)\right]\right\}
\end{aligned}
$$

Note that in deriving above formula (24), it was assumed that the vector quantities could be expanded everywhere. As a consequence the result contains the second derivative of $\mathbf{r}(t)$. However, its existence is not guaranteed for piecewise $C^{1}$ parametrization. Therefore, for formula (24) to be applicable, in general, an almost $C^{2}$ parametrization of $\mathrm{R}$ with is required. In the special case, where $\left\langle\mathbf{r}^{\prime}(t), \mathbf{v}(t)\right\rangle=0$ everywhere, the term containing $\mathbf{r}^{\prime \prime}(t)$ vanishes and a piecewise $C^{1}$ parametrization for the curves $\mathrm{R}$ and $\mathrm{B}$ is sufficient for a total twist formula to be applicable. In particular, this is true for a canonical ribbon.

\section{Writhe of polygonal curves}

An orientated line segment $s$ can be represented by a pair of vectors $(\mathbf{p}, \mathbf{a}) \in \mathbb{R}^{3} \times \mathbb{R}^{3}$ so that

$$
s=\{\mathbf{p}+u \mathbf{a} \mid u \in[0,1)\}
$$

A closed polygonal curve $R^{P}$ of length $\mathrm{N}$ is formed by $\mathrm{N}$ connected, non-intersecting line segments $s_{i} \equiv s_{i}\left(\mathbf{p}_{i}, \mathbf{a}_{i}\right)$, where the $\mathbf{p}_{i}$ are called the vertices. Then

$$
R^{P}=\bigcup_{i=1}^{N} s_{i}
$$

so that

1. The curve does not intersect itself $s_{i} \cap s_{j}=\emptyset$ for $i \neq j$

2. The curve is connected and closed $\mathbf{p}_{i}+\mathbf{a}_{i}=\mathbf{p}_{(i+1) \bmod N}$ 
Let $\hat{\mathbf{a}}_{i}=\left|\mathbf{a}_{i}\right|^{-1} \mathbf{a}_{i}$ and let $G_{i}$ be the geodesic on $S^{2}$ that connects $\hat{\mathbf{a}}_{i-1}$ and $\hat{\mathbf{a}}_{i}$. The tantrix of $R^{P}$ reads

$$
T_{R}=\bigcup_{i=1}^{N} G_{i}
$$

Let $\mathbf{d} \in \mathbb{R}^{3}$ so that $\hat{\mathbf{d}} \notin T_{R}$. A pushed off curve $R^{P}+\varepsilon \mathbf{d}$ is obtained by translating a copy of $R^{P}$ by the vector $\varepsilon \mathbf{d}$.

$$
R^{P}+\varepsilon \mathbf{d}=\bigcup_{i=1}^{N} s_{i}^{\prime}\left(\mathbf{p}_{i}+\varepsilon \mathbf{d}, \mathbf{a}_{i}\right)
$$

The parameter $\varepsilon>0$ must be chosen small enough, so that for all $\varepsilon^{\prime}$ with $0<$ $\varepsilon^{\prime} \leq \varepsilon\left(R^{P}+\varepsilon^{\prime} \mathbf{d}\right) \cap R^{P}=\emptyset$. The curves $R^{P}$ and $R^{P}+\varepsilon \mathbf{d}$ form a generalized ribbon with $\mathbf{v}(t)=\varepsilon \mathbf{d}=$ const. Therefore, the writhe of $R$ may be determined from the relation (11) as

$$
W r(R)=L k\left(R^{P}, R^{P}+\varepsilon \mathbf{d}\right)-T w\left(R^{P}+\varepsilon \mathbf{d}, R^{P}\right)
$$

However, it is not yet possible to apply the simple expression for the total twist equation (13), because the second derivative $\mathbf{r}^{\prime \prime}(t)$ is not defined near the vertices of the polygonal curve. Therefore, in order to determine $T w\left(R^{P}+\varepsilon \mathbf{d}, R^{P}\right)$, the edges at vertices shall be connected by quarter circles of small radius $\delta r$. The curve obtained by this smoothening procedure shall be called $R_{\delta r}$ and the limit $\delta r \rightarrow 0$ which recovers the polygonal curve $R \rightarrow R^{P}$ shall be called polygonal limit.

Given a parameter $\delta r$, consider a vertex $\mathbf{p}_{i}$ of $R^{P}$. The incident line segments form an inner angle $\bar{\alpha}_{i}=\pi-\alpha_{i}$, where

$$
\cos \alpha_{i}=\left\langle\hat{\mathbf{a}}_{i-1}, \hat{\mathbf{a}}_{i}\right\rangle
$$

As shown in figure 4, draw a line segment $w$ through the vertex $\mathbf{p}_{i}$, so that it divides the angle $\bar{\alpha}_{i}$ symmetrically. On w lies the point $\mathbf{C}_{i}$ which is defined so that its shortest distance to $s_{i}$ and $s_{i-1}$ is $\delta r$. Remove the pieces between the vertex $\mathbf{p}_{i}$ and the points of distance $\delta r$ from $\mathbf{C}_{i}$ from the segments $s_{i}$ and $s_{i+1}$. Reconnect the segments through a circle segment with center $\mathbf{C}_{i}$, radius $\delta r$ and length $\alpha \delta r$. Applying this procedure to all vertices of $R^{\text {Poly }}$ constructs the curve $R_{\delta r}$.

Let $l_{\delta r}$ be the length of $R_{\delta r}$ and let $u_{\delta r}=l_{\delta r} /(2 \pi)$. The curve $R_{\delta r}$ consists of straight segments on which the parametrization $\mathbf{r}(t)$ shall look as $\mathbf{r}(t) \sim u_{\delta r} t \hat{\mathbf{a}}$ and 


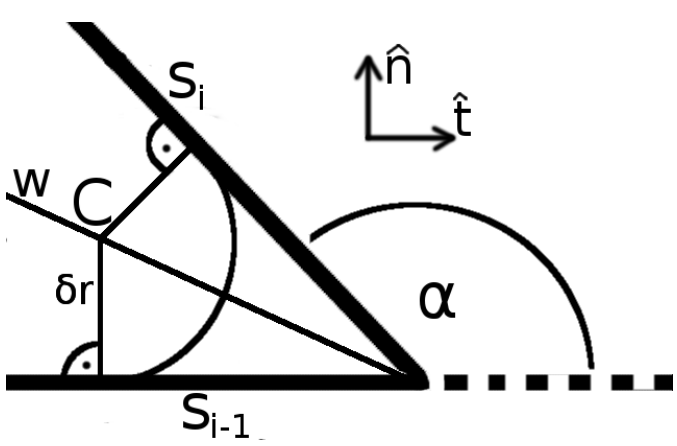

Fig. 4 The segments $s_{i-1}$ and $s_{i}$ form the angles $\alpha$ and $\bar{\alpha}=\pi-\alpha$. The constructed segment w divides the angle $\bar{\alpha}$ symmetrically. The point $\mathrm{C}$ is found on w at a distant $\delta r$ away from $s_{i}$. This point is the center of a circle of radius $\delta r$ inscribed in the wedge formed by the segments $s_{i}$ and $s_{i+1}$.

circle segments. Suppose the circle segment with center $\mathbf{C}_{i}$ is parametrized from the interval $\left[a_{i}, a_{i}+\delta t_{i}\right]$. Then, with $\hat{\mathbf{t}}_{i}=\hat{\mathbf{a}}_{i-1}$ and $\hat{\mathbf{n}_{i}}=\left(\sin \alpha_{i}\right)^{-1}\left(\hat{\mathbf{a}}_{i}-\left\langle\hat{\mathbf{a}}_{i}, \hat{\mathbf{t}}_{i}\right\rangle \hat{\mathbf{t}}_{i}\right)$ the parametrization of the segments reads

$$
\mathbf{r}_{i}(t)=\mathbf{C}_{i}+\delta r \sin \left(\frac{\alpha_{i}}{\delta t_{i}}\left(t-a_{i}\right)\right) \hat{\mathbf{t}}_{i}-\delta r \cos \left(\frac{\alpha_{i}}{\delta t_{i}}\left(t-a_{i}\right)\right) \hat{\mathbf{n}}_{i}
$$

where $\delta t_{i}$ is fixed by

$$
u_{\delta r}=\frac{\delta r \alpha_{i}}{\delta t_{i}}
$$

In order to determine $T w\left(R_{\delta r}+\varepsilon \mathbf{d}, R_{\delta r}\right)$, set $\mathbf{v}(t)=\varepsilon \mathbf{d}=$ const so that the rotation term in equation (13), which contains $\mathbf{v}^{\prime}$ vanishes. Only the acceleration term containing $\mathbf{r}^{\prime \prime}(t)$ survives. However, on straight segments $\mathbf{r}^{\prime \prime}(t)=0$, so that the source of generalized total twist comes from the circle segments only

$$
\begin{gathered}
T w=\frac{1}{2 \pi} \sum_{i} \int_{a_{i}}^{a_{i}+\delta t_{i}} I(t) \\
I(t)=\frac{\left|\mathbf{r}_{i}^{\prime}(t)\right|^{-1}\left\langle\mathbf{r}_{i}^{\prime}(t), \mathbf{d}\right\rangle}{\left|\mathbf{r}_{i}^{\prime}(t)\right|^{2} \mathbf{d}^{2}-\left\langle\mathbf{r}_{i}^{\prime}(t), \mathbf{d}\right\rangle^{2}}\left[\mathbf{d}, \mathbf{r}_{i}^{\prime}(t), \mathbf{r}_{i}^{\prime \prime}(t)\right]
\end{gathered}
$$

For the sake of brevity, drop the index i temporarily. Let $\hat{\mathbf{s}}=\hat{\mathbf{t}} \times \hat{\mathbf{n}}=\frac{\hat{\mathbf{a}}_{i-1} \times \hat{\mathbf{a}}_{i}}{\sin \alpha}$, so that $\hat{\mathbf{t}}, \hat{\mathbf{n}}, \hat{\mathbf{s}}$ are orthonormal to each other and $\mathbf{d}$ can be expressed as

$$
\mathbf{d}=d_{t} \hat{\mathbf{t}}+d_{n} \hat{\mathbf{n}}+d_{s} \hat{\mathbf{s}}
$$


Compute the terms relevant to the integrand

$$
\begin{aligned}
& \mathbf{r}^{\prime}(t)=u \cos \left(\frac{\alpha}{\delta t}(t-a)\right) \hat{\mathbf{t}}+u \sin \left(\frac{\alpha}{\delta t}(t-a)\right) \hat{\mathbf{n}} \\
& \mathbf{r}^{\prime \prime}(t)=-u \frac{\alpha}{\delta t} \sin \left(\frac{\alpha}{\delta t}(t-a)\right) \hat{\mathbf{t}}+u \frac{\alpha}{\delta t} \cos \left(\frac{\alpha}{\delta t}(t-a)\right) \hat{\mathbf{n}} \\
& \left\langle\mathbf{r}^{\prime}(t), \mathbf{d}\right\rangle=u d_{t} \cos \left(\frac{\alpha}{\delta t}(t-a)\right)+u d_{n} \sin \left(\frac{\alpha}{\delta t}(t-a)\right) \\
& {\left[\mathbf{d}, \mathbf{r}_{i}^{\prime}(t), \mathbf{r}_{i}^{\prime \prime}(t)\right]=u^{2} \frac{\alpha}{\delta t} d_{s}} \\
& \left|\mathbf{r}_{i}^{\prime}(t)\right|=u \\
& \left|\mathbf{r}_{i}^{\prime}(t)\right|^{2} \mathbf{d}^{2}-\left\langle\mathbf{r}_{i}^{\prime}(t), \mathbf{d}\right\rangle^{2}=u^{2}\left\{d_{t}^{2}+d_{n}^{2}+d_{s}^{2}\right. \\
& \left.-\left[d_{t} \cos \left(\frac{\alpha}{\delta t}(t-a)\right)+d_{n} \sin \left(\frac{\alpha}{\delta t}(t-a)\right)\right]^{2}\right\} \\
& =u^{2}\left(d_{s}^{2}+\left[d_{t} \sin \left(\frac{\alpha}{\delta t}(t-a)\right)-d_{n} \cos \left(\frac{\alpha}{\delta t}(t-a)\right)\right]^{2}\right)
\end{aligned}
$$

Combining equations (3-27) under the integral

$$
\begin{aligned}
\int_{a_{i}}^{a_{i}+\delta t_{i}} d t I(t)= & d_{s} \int_{a}^{a+\delta t} d t \frac{d_{t} \cos \left(\frac{\alpha}{\delta t}(t-a)\right)+d_{n} \sin \left(\frac{\alpha}{\delta t}(t-a)\right)}{d_{s}^{2}+\left[d_{t} \sin \left(\frac{\alpha}{\delta t}(t-a)\right)-d_{n} \cos \left(\frac{\alpha}{\delta t}(t-a)\right)\right]^{2}} \\
= & \frac{d_{s}}{d_{s}^{2}} \int_{a}^{a+\delta t} d t \frac{d_{t} \cos \left(\frac{\alpha}{\delta t}(t-a)\right)+d_{n} \sin \left(\frac{\alpha}{\delta t}(t-a)\right)}{1+\left[\frac{d_{t} \sin \left(\frac{\alpha}{\delta t}(t-a)\right)-d_{n} \cos \left(\frac{\alpha}{\delta t}(t-a)\right)}{\left|d_{s}\right|}\right]^{2}} \\
= & \frac{d_{s}}{\left|d_{s}\right|} \int_{-\frac{d_{n}}{\left|d_{s}\right|}}^{\frac{d_{t} \sin (\alpha)-d_{n} \cos (\alpha)}{\left|d_{s}\right|}} \frac{d x}{1+x^{2}} \\
= & \operatorname{sgn}\left(d_{s}\right)\left\{\arctan \left(\frac{d_{t} \sin (\alpha)-d_{n} \cos (\alpha)}{\left|d_{s}\right|}\right)\right. \\
& \left.+\arctan \left(\frac{d_{n}}{\left|d_{s}\right|}\right)\right\}
\end{aligned}
$$


Switching back to using the index $\mathrm{i}$, let $d_{i}:=\left\langle\mathbf{d}, \hat{\mathbf{a}}_{i}\right\rangle, d_{i}^{\perp}:=\frac{\hat{\mathbf{a}}_{i-1} \times \hat{\mathbf{a}}_{i}}{\sin \alpha_{i}}$ so that near $\mathbf{C}_{i}, d_{t}=d_{i-1}, d_{s}=d_{i}^{\perp}$ and $d_{n}=\left(\sin \alpha_{i}\right)^{-1}\left(d_{i}-d_{i-1} \cos \alpha_{i}\right)$. When the $d_{i}$ s are entered into eq (27), eq (26) reads

$$
\begin{aligned}
T w\left(R_{\delta r}+\varepsilon \mathbf{d}, R_{\delta r}\right)= & \frac{1}{2 \pi} \sum_{i=1}^{N} \operatorname{sgn}\left(d_{i}^{\perp}\right)\left\{\arctan \left(\frac{d_{i-1}-d_{i} \cos \alpha_{i}}{\left|d_{i}^{\perp}\right| \sin \alpha_{i}}\right)\right. \\
& \left.+\arctan \left(\frac{d_{i}-d_{i-1} \cos \alpha_{i}}{\left|d_{i}^{\perp}\right| \sin \alpha_{i}}\right)\right\}
\end{aligned}
$$

Note that all dependence on the parameter $\delta r$ has canceled so that the effect of the polygonal limit $\delta r \rightarrow 0$ is trivial, i.e.

$$
T w\left(R^{P}+\varepsilon \mathbf{d}, R^{P}\right)=T w\left(R_{\delta r}+\varepsilon \mathbf{d}, R_{\delta r}\right)
$$

This concludes the proof of lemma 3. With the conservation for generalized ribbons eq (11), it follows immediately from eq (29) that

$$
W r\left(R_{\delta r}\right)-W r\left(R^{\text {Poly }}\right)=L k\left(R_{\delta r}+\varepsilon \mathbf{d}, R_{\delta r}\right)-L k\left(R^{\text {Poly }}+\varepsilon \mathbf{d}, R^{\text {Poly }}\right)
$$

Therefore the difference between the writhe of a polygonal curve $R^{\text {Poly }}$ and its related curve $R_{\delta r}$ must be an integer. Note that the difference in linking number in eq (30) can only differ from zero, when the curve $R_{\delta} r$ passes through itself in the polygonal limit $\delta r \rightarrow 0$. However, $\delta r$ can always be chosen small enough so that one may say that for every polygonal curve exist radii $\delta r$ small enough so that the rounded off curve $R_{\delta r}$ has the same writhe as the polygonal curve.

\section{Lattice Ribbon}

This section deals with the proofs of lemmas 4 and 5. It is divided in three sub sections. The first subsection defines the lattice ribbon. In the second subsection lemma 4 is proved. Finally, lemma 5 is proved in the third subsection. There will be several references to the formula 13 of the generalized total twist. Henceforth, it shall be just referred to as twist integral. 
4.1 Definition of the Lattice Ribbon

The lattice ribbon $\mathscr{L}$ consists of two close avoiding curves on the simple cubic lattice $Z^{3}$. It can be constructed by using plaquettes. A plaquette $\rho$ is a unit square with integer vertices from which one constructs a closed ribbon $\mathscr{L}$ as an ordered sequence of plaquettes indexed by $i=1, . ., N$ such that

1. Plaquettes $\rho_{i}$ and $\rho_{i+1}$ which are adjacent in the sequence share a common edge. Also, $\rho_{1}$ and $\rho_{N}$ share a common edge. These shared edges $g_{i}=\rho_{i} \cap \rho_{i+1}$ are called ribbon edges. All other edges are called boundary edges.

2. No plaquette except $\rho_{i}$ and $\rho_{i+1}$ can share the edge $g_{i}^{(r i b)}=\rho_{i} \cap \rho_{i+1}$.

3. Only next- neighbor plaquettes can share only one vertex v. If $v=\rho_{i} \cap \rho_{j} \Rightarrow$ $|i-j|_{\operatorname{modN}}=2$.

4. When $|i-j|_{\bmod N}>2 \Rightarrow \rho_{i} \cap \rho_{j}=\emptyset$.

The boundary edges of the plaquettes of $\mathscr{L}$ form two lattice curves R, B. It shall also be required that the boundary curves $\mathrm{R}$ and $\mathrm{B}$ are closed. A plaquette is called an ordinary plaquette when it has one edge belonging to $R$ and one edge belonging to $B$. Otherwise a plaquette is called a $\mathrm{R}(\mathrm{B})$-corner plaquette, eg it has two edges belonging to R (B). Note, that it follows from conditions (2) and (3) that no two corner plaquettes of the same label can be adjacent.

Every plaquette $\rho_{i}$ induces a center curve $C$ starting at the midpoint of $g_{i-1}^{(r i b)}$, stepping to the barycenter of the plaquette and ending on the midpoint of $g_{i}^{(r i b)}$. Corresponding to the steps of the center curve associate two vectors

$$
\begin{aligned}
& c_{1}\left(\rho_{i}\right)=\operatorname{Center}\left(\rho_{i}\right)-\operatorname{Center}\left(e_{i-1}^{(r i b)}\right) \\
& c_{2}\left(\rho_{i}\right)=\operatorname{Center}\left(e_{i}^{(r i b)}\right)-\operatorname{Center}\left(\rho_{i}\right)
\end{aligned}
$$

of length $1 / 2$ with the plaquette $\rho_{i}$.

\subsection{Total twist of the lattice ribbon}

The lattice ribbon $\mathscr{L}$ is bounded by the curves $R$ and $B$. We are interested in deriving an algorithm to determine the total twist with respect to $R$. The curve $R_{\delta r}$ is obtained 
from $R$ by connecting edges at corners of $R$ through quarter circles of radius $\delta r$. As described in the previous section, this automatically induces a $C^{1}$ parametrization $\mathbf{r}(t)$ of $R_{\delta r}$ with

$$
\left|\mathbf{r}^{\prime}(t)\right|=u_{\delta r}^{R}
$$

where $u_{\delta r}^{R}=l\left(R_{\delta r}\right) /(2 \pi)$ is the constant parametrization speed, which depends on the length $l\left(R_{\delta r}\right)$ of the curve $R_{\delta r}$. In combination with the polygonal limit, the parametrization $\mathbf{r}(t)$ of $R_{\delta r}$ can be used in the twist integral to determine

$$
T w(B, R)=\lim _{\delta r \rightarrow 0} T w\left(B, R_{\delta r}\right)
$$

In order to compute eq (31), we also require a piecewise $C^{1}$ parametrization of a vector field $V_{\delta r}$ which points from $R_{\delta r}$ onto $B$, covering both. In practice, $V_{\delta r}$ can be defined through $\mathbf{v}(t)=\mathbf{b}(t)-\mathbf{r}(t)$, i.e. by providing a piecewise $C^{1}$ parametrization of $B . B$ will be parametrized linearly everywhere with a parametrization speed that is may jump between finitely many intervals $I$. Thus, there are many choices to define the vector field $V_{\delta r}$ and the proof basically consists of making a particular choice. We denote the generalized ribbon formed by $\left(R_{\delta r}, V_{\delta r}\right)$ by $\mathscr{G}_{\delta r}$, and the generalized ribbon obtained from $\mathscr{G}_{\delta r}$ in the polygonal limit by $\mathscr{G}$. We define a section $S$ of $\mathscr{G}_{\delta r}$ as a piece of the curve $R_{\delta r}$ and the corresponding vector field $V_{\delta r}$ on it. By dividing $\mathscr{G}_{\delta r}$ into connected sections, we may write

$$
\begin{aligned}
\mathscr{G}_{\delta r} & =\cup_{i} S_{i} \\
T w(B, R) & =\sum_{i} \lim _{\delta r \rightarrow 0} T w\left(S_{i} \mid R_{\delta r}\right)
\end{aligned}
$$

where $T w\left(S_{i} \mid R_{\delta r}\right)$ is the twist with respect to $R_{\delta r}$ of section $S_{i}$. This quantity can be computed from the twist integral by changing the integral boundaries to the parametrization time of the corresponding section. The two vectors that bound a section $S$ shall be denoted $\mathbf{V}_{a}(S)$ and $\mathbf{V}_{b}(S)$.

We describe a method where the underlying lattice ribbon induces the vector field $V_{\delta r}$ on $R_{\delta r}$, so that the sections for which $\lim _{\delta r \rightarrow 0} T w\left(S \mid R_{\delta r}\right) \neq 0$ can be traced back to a few predefined configurations of plaquettes. These configurations may then be regarded as the source of the twist. 


\subsubsection{Sequence and Section}

An ordered sequence of connected plaquettes $\left\{\rho_{1}, \rho_{2}, \ldots, \rho_{n}\right\} \in \mathscr{L}$ with $n>0$ shall be called a sequence $s$. In addition to the two boundary curves $R$ and $B$, a sequence is bounded by ribbon edges $g_{a} \in \rho_{1}$ and $g_{b} \in \rho_{n}$. Denote the first and last point in $s$ which lie on the curve $R$ by $\mathbf{R}_{a}[s]$ and $\mathbf{R}_{b}[s]$. The analogous points on the curve $B$ shall be denoted by $\mathbf{B}_{a}[s]$ and $\mathbf{B}_{b}[s]$. Then, the vectors

$$
\begin{aligned}
& \mathbf{V}_{a}[s]=\mathbf{B}_{a}[s]-\mathbf{R}_{a}[s] \\
& \mathbf{V}_{b}[s]=\mathbf{B}_{b}[s]-\mathbf{R}_{b}[s]
\end{aligned}
$$

point along the ribbon edge $g_{a}$ and $g_{b}$, respectively.

Any division of a lattice $\mathscr{L}=\left\{\rho_{1}, \ldots, \rho_{N}\right\}$ into connected, non-overlapping sections $s_{i}$ can be regarded as a sketch of a generalized ribbon $\mathscr{G}$. By this we mean that a division into sequences defines vectors $\mathbf{V}_{a}[s]$ at points $\mathbf{R}_{a}[s]$ along the lattice curve $R$. These may be regarded as part of the vector field $V$, that defines the generalized ribbon $\mathscr{G}$. However, these few points do not define $V$ completely. $V$ is merely sketched.

The division of the lattice ribbon also defines a sketch of a ribbon $\mathscr{G}_{\delta r}$ with boundary curves $R_{\delta r}$ and $B$ and vector field $V_{\delta r}$. The divisions of the ribbon $\mathscr{G}_{\delta r}$ are called sections $S$ and we will distinguish two kind of sections.

A ribbon edge section (RES) $S^{-}(s)$ is induced near a ribbon edge $g_{a}=s^{\prime} \cap s$ at which two sequences $s^{\prime}$ and $s$ join. The RES consists of all points on $R_{\delta r}$ that lie within a distant $\delta r$ of $\mathbf{R}_{a}[s]$. Also, all parts of $B$ that lie within a distant $\delta r$ from $\mathbf{B}_{b}[s]$ belong to the RES. The vector field $V_{\delta r}$ on the RES shall be defined by its parametrization as follows. Suppose, the parts of $R_{\delta r}$ on the RES are parametrized from the interval $\left[a_{S}, b_{S}\right]$, then parametrize the parts of $B$ on the RES from the same interval with constant parametrization speed

$$
u_{R E S}^{B}= \begin{cases}4 \pi^{-1} u_{\delta r}^{R} & \text { if } \mathbf{R}_{a} \text { is a corner vertex } \\ u_{\delta r}^{R} & \text { otherwise }\end{cases}
$$


Then, $V_{\delta r}$ on the RES is defined by $\mathbf{v}(t)=\mathbf{b}(t)-\mathbf{r}(t), t \in\left[a_{S^{-}}, b_{S^{-}}\right]$. The outer vectors of the vector field $V_{\delta}$ on a section are denoted by $\mathbf{V}_{a}[S]$ and $\mathbf{V}_{b}[S]$. In this case they read

$$
\begin{aligned}
\mathbf{V}_{a}\left[S^{-}(s)\right] & =\mathbf{v}\left(a_{S^{-}}\right) \\
\mathbf{V}_{b}\left[S^{-}(s)\right] & =\mathbf{v}\left(b_{S^{-}}\right)
\end{aligned}
$$

In the polygonal limit these vectors converge, from opposite sides, towards the vector $\mathbf{V}_{a}[s]$ which lies along the ribbon edge. Note that the notation of a RES $S^{-}(s)$ contains a minus as superscript. This is meant to indicate that the RES is found near the first ribbon edge $\rho_{a}$ of the sequence $s$. A second ribbon edge section is found near the end of $s$. This RES shall then be denoted $S^{+}(s)$. Of course, when $s_{k}$ and $s_{k+1}$ are two consecutive sequences $S^{+}\left(s_{k}\right)$ and $S^{-}\left(s_{k+1}\right)$ identify the same section.

The second type of section shall be called sequence induced section (SIS). A sequence $s$ induces a SIS, that is bounded by the sections $S^{-}(s)$ and $S^{+}(s)$. Any choice of parametrization $\mathbf{b}(t)$ of $B$ on the interval $\left[b_{S^{-}}, a_{S^{+}}\right]$with $\mathbf{v}\left(b_{S^{-}}\right)=\mathbf{V}_{b}\left[S^{-}(s)\right]$ and $\mathbf{v}\left(a_{S^{+}}\right)=\mathbf{V}_{a}\left[S^{+}(s)\right]$ can be used to define the twist of the underlying sequence $s$ as

$$
T w(s)=\lim _{\delta r \rightarrow 0} T w\left(S(s) \mid R_{\delta r}\right)
$$

First, note from the twist integral that when all points of $R_{\delta r}$ and $B$ of any section $S$ lie in a plane the twist vanishes. These sections shall be called planar. In particular a sequence that lies in a plane induces a planar section so that $T w(s)=0$. Such a sequence shall be called planar sequence.

Second, the twist integral and thus the twist of a sequence and a section is invariant under the first three of the following symmetry transformations

1. Translation by a vector $\mathbf{d} \in \mathbb{Z}^{3}: s^{\prime}=s+\mathbf{d}$

2. Rotation: A rotation of $s$ by $\pi / 2$ around any of the coordinate axis

3. Reversal: $s^{\prime}=\left\{\rho_{n}, . ., \rho_{2}, \rho_{1}\right\}$

4. Reflection: reflection at one of the coordinate planes

Under the fourth transformation, reflections, the twist picks up a sign due to the triple product structure of the twist integral. The symmetry transformations can be used 
to define an equivalence class $C$ called configuration. Two sequences $s_{1}$ and $s_{2}$ are equivalent if $s_{1}$ can be transformed into $s_{2}$ by any combination of the transformations (1-4). The configuration class can be split into two sub classes of equivalence denoted as $C^{r}$ and $C^{l}$, where $C^{r}$ contains the sequences with $T w(s)>0$. The reflection symmetry transforms between the helical sub classes. We define

$$
T w(C)=T w(s)
$$

for any sequence $s \in C^{r}$. When $C^{r}=C^{l}, T w(C)=0$ and the configuration has no helicity.

\subsubsection{A basic set of configurations}

With a division of the ribbon into sequences $s_{k}$, eq (32) can be written as

$$
T w(B, R)=\sum_{k} T w\left(s_{k}\right)+\sum_{k} T w\left(S^{+}\left(s_{k}\right)\right)
$$

The goal is to have a set of configurations $C_{i} i=1, . ., N_{C}$ and to find a division of the lattice ribbon into sequences $s_{k}$ so that either $s_{k} \in C_{i}$ for some i or $s_{k}$ is planar. It shall also be required that $S^{+}\left(s_{k}\right)=0$ for all $k$. When we also count by $n_{i}^{r / l}$ how often a sequence of configuration $C_{i}$ with right/left-handed helicity is found in the ribbon, the twist of such a division is given by

$$
T w(B, R)=\sum_{i}^{N_{C}}\left(n_{i}^{r}-n_{i}^{l}\right) T w\left(C_{i}\right)
$$

To see what happens in general, consider the lattice ribbon $\mathscr{L}=\left\{\rho_{1}, \ldots, \rho_{6}\right\}$ shown in figure 5. Its twist is $T w(B, R)=0$. However, when we divide it into three sequences $s_{k}$ of two plaquettes each so that $\mathscr{L}=s_{1} \cup s_{2} \cup s_{3}$, we find that

$$
T w(s) \neq \sum_{k} T w\left(s_{k}\right)=1 / 4
$$

Note the arrows from the curve $R$ onto $B$ drawn into figure 5. These represent parts of the vector field $V$ on $R$. Therefore, the arrows represent a sketch of a ribbon $\mathscr{G}$. This sketch is induced by the division of $\mathscr{L}$ into the sequences $s_{k}$.

While the sequences $s_{1}$ and $s_{3}$ yield no twist, $T w\left(s_{2}\right)=1 / 4$. The unaccounted twist difference can be associated with the ribbon edges between the sections. There 


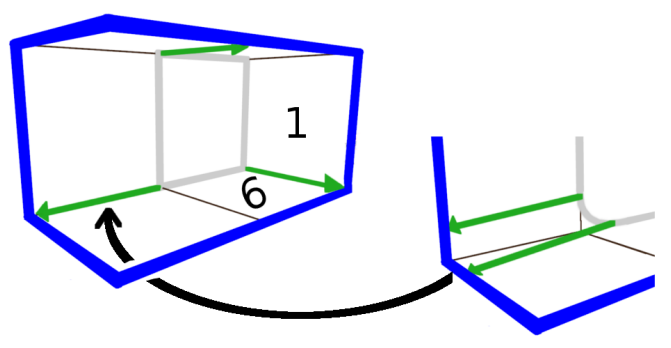

Fig. 5 The depicted lattice ribbon $\mathscr{L}$ is formed by six plaquettes with the first and last plaquette labeled by number. The lattice ribbon is divided into three sequences, each two plaquettes long. This sketches the ribbon $\mathscr{G}$ as indicated by the arrows along the ribbon edges between the sequences. The rhs shows a sketch of a RES of $\mathscr{G}_{\delta r}$. In the polygonal limit the ribbon edge section, bounded by the shown arrows, collapses into the vector along the ribbon edge. This particular ribbon edge is problematic because the twist of the RES does not vanish in the polygonal limit.

are three such ribbon edges in our example: $g_{1}=s_{1} \cap s_{2}, g_{2}=s_{2} \cap s_{3}, g_{3}=s_{3} \cap$ $s_{1}$. With the parameterizations (eq.(33)) of the RES, it can be shown with the twist integral, that there is no twist from the RESs at $g_{1}$ and $g_{3}$ in the polygonal limit, i.e. $\lim _{\delta r \rightarrow 0} T w\left(S^{+}\left(s_{1 / 3}\right) \mid R_{\delta r}\right)=0$. For the RES at $g_{2}$ the twist integral produces a diverging result in the polygonal limit. This limit is inicated on the right-hand side of figure 5. The figure shows the outer vectors of the RES section collapse into the vector $\mathbf{V}_{a}\left[s_{3}\right]$. However, note that this vector is aligned with one of the two tangents to $R$ at $\mathbf{R}_{a}\left[s_{3}\right]$. Thus, our paramaetrization has not produced a proper generalized ribbon. We shall label all ribbon edges at which this may potentially occur as problematic. A RES at a problematic ribbon edge shall be called a problematic RES.

Table 1 shows all problematic ribbon edges. RES at ribbon edges that are not problematic yield no twist in the polygonal limit.

We want to find a basic set of configurations and an algorithm that divides the lattice ribbon into sequences $s \in C_{i}$ and planar sequences, so that the lattice ribbon is never divided along a problematic edge. In order to keep the basic set as simple as possible, this will be achieved by introducing half sized plaquettes.

1. Every ordinary plaquette $\rho$ is divided in the middle. Thereby, introducing a ribbon edge that connects the middle of the $R$ edge with the middle of the $B$ edge. Denote 
the two emerging half sized plaquettes by $\rho_{a}$ and $\rho_{b}$. When the lattice ribbon is split into sequences at one of these new ribbon edges, the induced RES is planar and yields no contribution to twist in the polygonal limit.

2. Every $B$ corner plaquette $\rho$, whose two neighboring plaquettes lie in the same plane as $\rho$ is split along the diagonal. The new ribbon edge connects the midpoint of $B$ on this plaquette with the $R$ vertex across. We shall address the emerging half sized plaquettes as $\rho_{a}$ and $\rho_{b}$. Note that a corresponding induced RES is planar and thus twist free.

The diagrams in table 2 define our basic set of configurations. The twist values of a configuration can be computed via the twist integral. In the appendix such a computation is performed for configuration $C_{6}$. Alternatively, it suffices to consider how much $\mathbf{V}_{a}$ has to be twisted around $R$ to obtain $\mathbf{V}_{b}$.

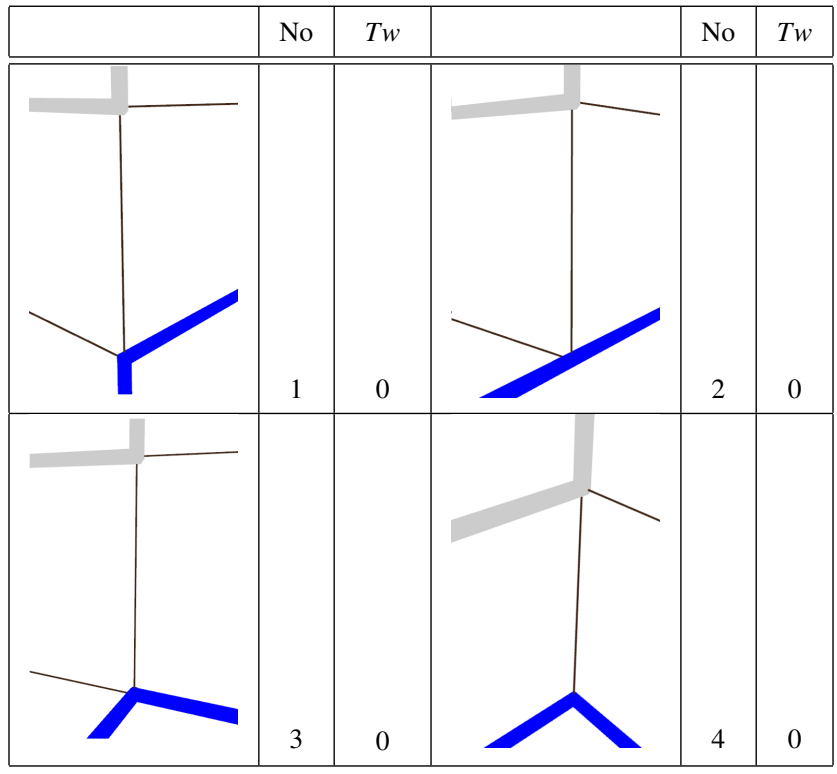

Table 1 The table shows parts of a curve $R$ (light) and $B$ (dark) near problematic ribbon edges. Characteristic of problematic ribbon edges is that an arrow drawn along the ribbon edge, from the $R$ vertex onto the $B$ vertex is tangent to $R$. The exceptions are planar ribbon edges. 


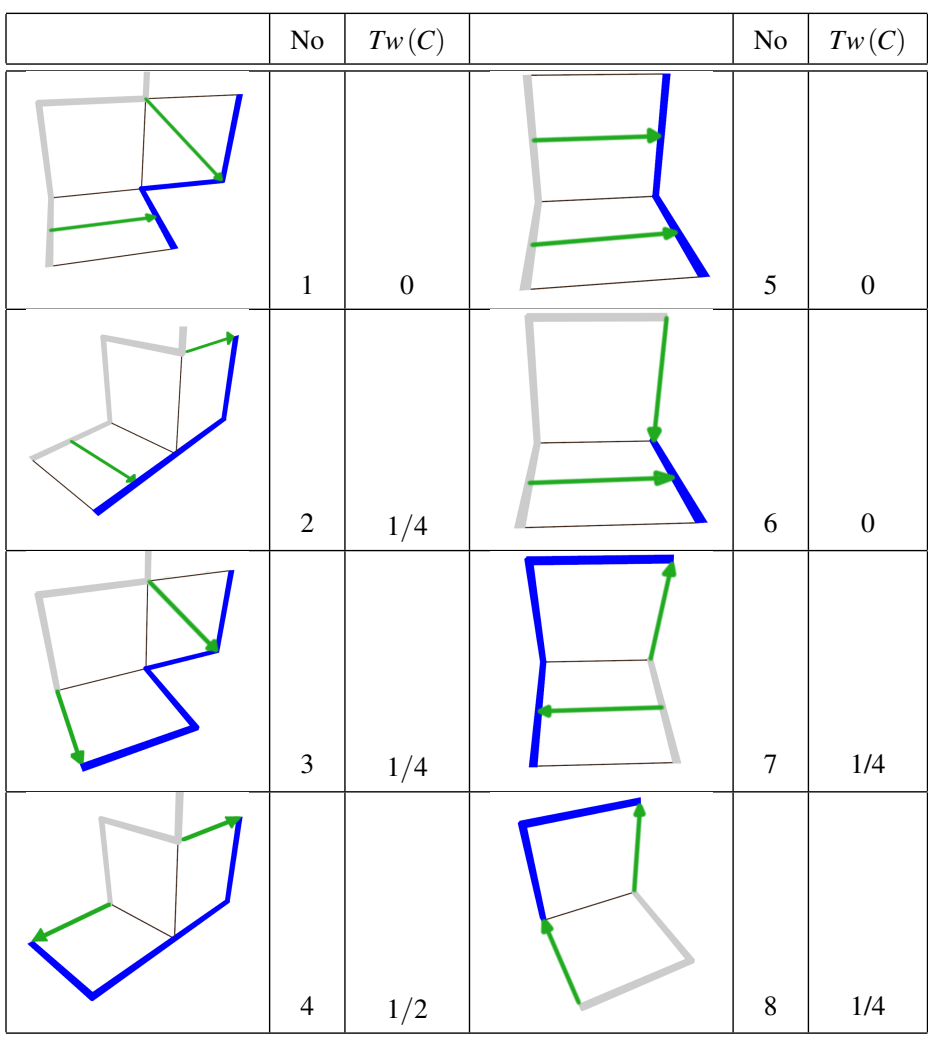

Table 2 The sequences of plaquettes shown in the diagrams define eight equivalence classes of configurations. The curve $R$ is shown in light-, the curve $B$ in dark. Every configuration is numbered and has an associated twist. We distingish between full and half-sized plaquettes. The arrows border the configuration. The configurations on the left are incident on three different full sized plaquettes. In addition, the diagrams show a piece of a fourth full plaquette. Although this fourth plaquette is not part of the configuration itself, the configuration is only identified when the fourth plaquette is aligned as indicated.

\subsubsection{Algorithm for extracting the twist}

With the basic configuration set defined, any lattice ribbon $\mathscr{L}$ can be divided by the following procedure. Divide every ordinary plaquette of $\mathscr{L}$ in the middle to produce additional ribbon edges as well as additional plaquettes. Also, divide $B$ corner plaquettes whose neighbors lie in the same plane. Plaquettes that retain their original size shall be adressed specifically as full plaquettes when the distinction is required. 
Pick an ordinary plaquette (there must be four at least) and start at the inner ribbon edge that divides the plaquette. Choose an orientation and label the plaquettes by index so that $\rho_{i}$ and $\rho_{i+1}$ share a ribbon edge $g$.

Traverse the lattice ribbon until reaching a ribbon edge at which the two incident plaquettes are perpendicular to each other. Analyze if plaquettes near that ribbon edge form a configuration $C_{i} i=1, . ., 4$. When more than one configuration is found pick the configuration whose first plaquette has the smallest index. If no configuration is formed, the two plaquettes form a configuration $C_{i} i=5, . ., 8$. All plaquettes that form the discovered configuration shall be marked as a sequence $s$ of the division of $\mathscr{L}$. Proceed to traverse the ribbon starting at the ribbon edge incident on the marked plaquette with the highest index. Now however, when a ribbon edge is reached, plaquettes that are marked may not be considered in the formation of configurations a second time. Count by $n_{i}^{r / l}$ how often the configuration $C_{i}$ was identified with helicity $r / l$.

This algorithm (with the choice of basic configurations) has the following properties

1. When $\rho_{k}$ is a plaquette that remains unmarked, move against the ribbon orientation until a marked plaquette $\rho_{k-m-1}$ is encountered. Afterwards, move to the other side until a marked plaquette $\rho_{k+p+1}$ is reached. Then, the sequence $\left\{\rho_{k-m}, \ldots, \rho_{k}, \ldots \rho_{k+p}\right\}$ is planar.

2. No $B$ corner plaquette which has a perpendicular neighbor is part of a planar sequence. (This is not true for $R$ corner plaquettes)

3. The algorithm does not divide the ribbon at problematic ribbon edges.

While property 1 is trivial, the other two can be shown by considering the combinatorics.

In order to show property 2 , one considers that a full $B$ corner plaquette $\sigma$ is attached at the end of planar sequence. Then, assume on the other side the plaquette $\tau$ is attached perpendicularly to $\sigma$. By considering the behavior of the algorithm as it approaches the ribbon edge $g=\sigma \cap \tau$ from both sides, while taking into account the 
possibilities for the neigboring plaquettes of $\tau: \tau^{\prime}, \tau^{\prime \prime}, \ldots$, one finds that $\tau$ and $\sigma$ must be part of the same sequence.

Property 3 is shown by considering the behaviour of the algorithm when it approaches a problematic ribbon edge formed between the plaquettes $\sigma$ and $\tau$. Considering all possibilities one concludes that $\sigma$ and $\tau$ must be part of the same sequence. Note that in addition to the configurations $C_{1-4}$, it is necessary to consider the problematic ribbon edge formed when $\sigma$ is an $R$ corner plaquette perpendicular to the $B$ corner plaquette $\tau$. Their neighbors $\sigma^{\prime}$ and $\tau^{\prime}$ must lie in the same plane as $\sigma$ and $\tau$, respectively. Another problematic ribbon edge is formed when an ordinary plaquette $\sigma$ is attached to a perpendicular $B$ corner plaquette $\tau$, and $\tau^{\prime}$ lies in the same plane as $\tau$.

In consequence of the properties, the twist is given by eq (34) and every full $B$ corner plaquette which has at least one perpendicular neighbor is part of a sequence that forms one of the configurations $C_{2}, C_{3}, C_{4}, C_{7}$ or $C_{8}$. But, these configurations are the only configurations that have twist so that by considering them and their twist value, we can conclude lemma 4.

Lemma 4 shall be formulated in an alternative form. Denote the set of all R (B) corner plaquettes of $\mathscr{L}$ by $\mathscr{L}_{R(B)}$.

With

$$
\operatorname{sgn}(x)= \begin{cases}0 & x=0 \\ 1 & x>0 \\ -1 & x<0\end{cases}
$$

the total twist of a lattice ribbon reads

$$
\begin{aligned}
& T w(B, R)=\frac{1}{4} \sum_{\rho_{i} \in \mathscr{L}_{B}} \operatorname{sgn}\left\{\left[c_{2}\left(\rho_{i-1}\right)+c_{1}\left(\rho_{i+1}\right), c_{1}\left(\rho_{i}\right), c_{2}\left(\rho_{i}\right)\right]\right\} \\
& T w(R, B)=\frac{1}{4} \sum_{\rho_{i} \in \mathscr{L}_{R}} \operatorname{sgn}\left\{\left[c_{2}\left(\rho_{i-1}\right)+c_{1}\left(\rho_{i+1}\right), c_{1}\left(\rho_{i}\right), c_{2}\left(\rho_{i}\right)\right]\right\}
\end{aligned}
$$

This formulation is equivalent to lemma 4 , when it is noted that by the construction rules of the ribbon $c_{2}\left(\rho_{i-1}\right)+c_{1}\left(\rho_{i+1}\right) \neq 0$ is implied as long as $\rho_{i}$ is a corner plaquette. The sign of the triple product of $c_{1}\left(\rho_{i}\right), c_{2}\left(\rho_{i}\right)$ with a neighboring center curve vector determines weather a left- or right-handed configuration is formed. 
4.3 Writhe of the center curve
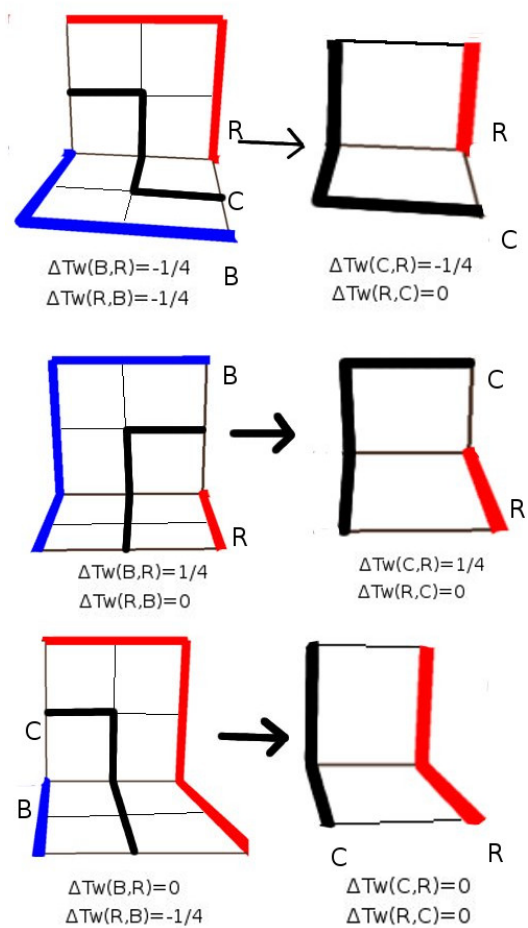

Fig. 6 The left-hand side shows all configurations (modulus symmetry transformations) which contribute to either $T w(B, R)$ or $T w(R, B)$, or both. It also shows the center curve $C$ on the plaquettes. Also, the grid of the half integer lattice is drawn. The center curve $C$ and the curve $R$ form a lattice ribbon on the half integer lattice. The right-hand side shows relevant two plaquette configurations of this ribbon that emerge from the configuration on the left. One finds that a configuration that yields twist with respect to $R$ of the original ribbon, produces a configuration of the half integer ribbon that yields twist with respect to $R$. However, it has no twist with respect to $C$. Every configuration that does not yield twist with respect to $R$, produces a configuration that yields neither twist w.r.t. $R$ nor to $C$.

The above result regarding which configurations of plaquettes are responsible for the twist can be used to prove lemma 5 on the center curve $\mathrm{C}$ of a lattice ribbon. It states 
that for a closed lattice ribbon with boundary curves $R$ and $B$

$$
W r(C)=L k(R, B)
$$

Proof The center curve $C$ and the boundary curve $R$ form a new lattice ribbon on the half integer lattice. One finds (figure 6) that there is no configuration of the half integer ribbon that produces twist with respect to to $C$ so that $T w(R, C)=0$. Also, every configuration of the original ribbon that contributes twist with respect to $R$, turns into a configuration of the half integer ribbon that contributes the same amount of twist to $T w(C, R)$, so that $T w(C, R)=T w(B, R)$. Using the conservation relation for generalized ribbons

$$
\begin{aligned}
W r(C) & =L k(R, C)-T w(R, C) \\
& =L k(R, C) \\
& =W r(R)+T w(C, R) \\
& =L k(B, R)
\end{aligned}
$$

\section{Acknowledgments}

One of the authors, ED, gratefully acknowledges the financial support of the University of Melbourne via its Melbourne International Research Scholarships scheme. Financial support from the Australian Research Council via its support for the Centre of Excellence for Mathematics and Statistics of Complex Systems and the Discovery Projects scheme is gratefully acknowledged by one of the authors, ALO.

\section{References}

1. Pankaj K. Agarwal, H. Edelsbrunner, and Y. Wang. Computing the writhing number of a polygonal knot. Discrete and Computational Geometry, 32:37-53, 2004.

2. J. Aldinger, I. Klapper, and M. Tabor. Formulae for the calculation and estimation of writhe. Journal of Knot Theory and Its Ramifications, 32:37-53, 1995.

3. G. Câlugâreanu. L'intègrale de gauss et l'analyse des noeuds tridimensionnels. Rev. Math. Pures Appl, 4:5-20, 1959. 
4. G. Câlugâreanu. Sur les classes d'isotopie des noeuds tridimensionnels et leurs invariants. Czech. Math. J, 11:588-625, 1961.

5. G. Câlugâreanu. Sur les enlacements tridimensionnels des courbes fermées. Comm. Acad. R. P. Romîne, 11:829-832, 1961.

6. J. Cantarella. On comparing the writhe of a smooth curve to the writhe of an inscribed polygon. SIAM Journal on numerical analysis, 42(5):1846 - 1861, 2004.

7. M. H. Eggar. On White's formula. Journal of Knot Theory and its Ramifications, 9(5):611 - 615, 2000.

8. F. B. Fuller. The writhing number of a space curve. Proc Natl Acad Sci U S A, 68:815-819, 1971.

9. F. B. Fuller. Decomposition of the linking number of a closed ribbon: A problem from molecular biology. Proceedings of the National Academy of Sciences, 75(8):3557-3561, 1978.

10. E. Janse van Rensburg, E. Orlandini, D. W. Sumners, M. Tesi, and S. Whittington. Entanglement complexity of lattice ribbons. Journal of Statistical Physics, 85:103-130, 1996.

11. E. Janse van Rensburg, E. Orlandini, D. W. Sumners, M. C. Tesi, and S. G. Whittington. Lattice ribbons: A model of double-stranded polymers. Phys. Rev. E, 50:4279-4282, 1994.

12. K. Klenin and J. Langowski. Computation of writhe in modeling of supercoiled DNA. Biopolymers, 54:307-317, Jan 2000.

13. E. Orlandini and E. Janse van Rensburg. Twist in an exactly solvable directed lattice ribbon. Journal of Statistical Physics, 80:781-791, 1995.

14. W. F. Pohl. DNA and differential geometry. Math. Intell., 3:20-27, 1980.

15. R. L. Ricca and B. Nipoti. Gauss' linking number revisited. Journal of Knot Theory and Its Ramifications, 20(10):1325-1343, 2011.

16. J. H. White. Self-linking and the Gauss integral in higher dimensions. American Journal of Mathematics, 91(3):pp. 693-728, 1969. 


\section{Appendix}

\section{A SAPs as center curves of lattice ribbons}

In this section of the appendix, we discuss the properties of SAPs which can be center curves of a lattice ribbon. The center curve $C$ of a lattice ribbon lies on the half integer simple cubic lattice.

We can distinguish two properties of $C$, a local property, and a closing condition. The closing condition guarantees that the boundaries are formed by two different SAP (ie. The ribbon is orientable).

Rather than lying on the half integer lattice, suppose $C$ is a SAP on the regular (integer) simple cubic lattice. Then, the size of a plaquettes is $L \times L$, where $L=2 k^{-1}$ with $k=1$. The parameter $k \in \mathbb{N}$ was introduced to generalize the plaquette size, so that that the results apply to lattice ribbons with rescaled plaquettes. Denote the orientation of a plaquette $\sigma$ by its normal vector $\hat{\sigma}$. Suppose the SAP is formed by $N_{s}$ segments $s$. Denote the orientation of a segment by $\hat{s}$.

Suppose two segments $s_{1}$ and $s_{2}$ of $C$ meet at a vertex $v=s_{1} \cap s_{2}$. Then there are two possibilities for plaquettes to be orientated around $v$.

1. $v$ is the center of a corner plaquette. Then, all plaquettes on the segments $s_{1}$ and $s_{2}$ have the same orientation given by $\hat{s}_{1} \times \hat{s}_{2}$

2. $v$ is the center of a ribbon edge between two plaquettes. In this case, the plaquettes on $s_{1}$ have orientation $\hat{s}_{2}$ and the plaquettes on $s_{2}$ have orientation $\hat{s}_{1}$

Denote the length of a segment $s$ by $l(s)$. In order to fit plaquettes onto the segment the following condition has to be met

$$
l(s)=n_{C}(s) k^{-1}+n_{O}(s) 2 k^{-1}
$$

where $n_{C}=0,1,2$ is the number of corner plaquettes on the segment $s$ and $n_{O}$ denotes the number of ordinary plaquettes fitted onto the line segment. Note that corner plaquettes can be found only at the beginning or the end of a segment so there can be no more than two. Corner plaquettes contribute one half of there center line to the length of different segments so there length contribution to one segment is $L / 2=k^{-1}$

Consecutive segments of center curves of lattice ribbons have to meet condition 35 and the condition on possible plaquette orientation at the same time. For example, the following constellation can not be part of the center curve of a lattice ribbon.

Consider three consequtive segments $s_{1} s_{2} s_{3}$ with orientations $\left[\hat{s}_{1}, \hat{s}_{2}, \hat{s}_{3}\right] \neq 0$ and lengths $l_{i}=l\left(s_{i}\right)$, so that $k l_{1}$ and $k l_{3}$ are odd and $k l_{2}$ even. Because of condition (eq. 35) $s_{1}$ and $s_{3}$ must have exactly one corner plaquette, whereas $s_{2}$ may have either 0 or 2 corner plaquettes. There are two possibilities for the position of the corner plaquette on $s_{1}$

1. The corner plaquette is on the vertex $s_{1} \cap s_{2}$. Then, $s_{2}$ must have two corner plaquettes because it cannot have only one. Therefore, the corner plaquette on $s_{3}$ is the same as the second corner plaquette of $s_{2}$. The plaquettes on $s_{2}$ must have orientations $\hat{s}_{1} \times \hat{s}_{2}$ and at the same time $\hat{s}_{2} \times \hat{s}_{3}$ so that one finds

$$
\hat{s}_{1} \times \hat{s}_{2}=\hat{s}_{2} \times \hat{s}_{3}
$$


which contradicts the setup $\left[\hat{s}_{1}, \hat{s}_{2}, \hat{s}_{3}\right] \neq 0$.

2. There is no corner plaquette on the vertex $s_{1} \cap s_{2}$. Consequently, $s_{2}$ cannot have any corner plaquettes, so there is no corner plaquette at $s_{2} \cap s_{3}$. The implies that the plaquettes on $s_{2}$ are orientated as $\hat{s}_{1}$ and at the same time as $\hat{s}_{3}$. So that

$$
\hat{s}_{1}=\hat{s}_{3}
$$

which again contradicts the setup.

Even when the SAP satisfies the local conditions discussed above, it may only admit a non-orientable lattice ribbon. A non-orientable lattice ribbon possesses only one boundary curve so that linking number is not defined. Nevertheless, it is possible to make sense of linking number. Cut an ordinary plaquette $\sigma$ perpendicular to the boundary edges. Label the cutting points of the boundary edges $R_{0}$ and $B_{0}$. As the ribbon is traversed in one direction the boundaries are labeled either $R$ or $B$. When the cutting point is neared, the $R$ boundary is about to connect to $B_{0}$ and $B$ is about to connect with $R_{0}$. Instead of making the connection, connect the end of $B$ across the plaquette onto $B_{0}$ and likewise for $R$. Therefore, the two curves $R$ and $B$ are closed and linking number is defined. Linking number can be determined from a projection diagram as one half of the sum of all signed crossings. When we choose a projection diagram in the direction $\hat{\sigma}+\varepsilon(1,1,1)$, one can make sure by choosing the cutting point, that the cross connection of the curves do not produce any signed crossings except the one which is due to the cross connections passing over each other. This crossing contributes $1 / 2$ to the linking number. Therefore, if we had not made the cross connection, determining the linking number from a projection diagram yields $L k=n+1 / 2$ with some $n \in \mathbb{Z}$. Assuming that the relation $\operatorname{Lk}(B, R)=W r(C)$ extends to the broader definition of linking number, one may conclude that a SAP $C$ that admits a non-orientable ribbon has writhe $W r(C)=n+1 / 2$.

\section{B Computing the twist of a configuration}

As an example, we compute the twist of a sequence $s \in C_{6}$, by defining a section on $R_{\delta r}$. The section can be defined by providing a parametrization $\mathbf{b}(t)$ relative to $\mathbf{r}(t)$. Suppose, the section is parametrized from the interval $I_{S}=\left[t_{a}, t_{b}\right]$ and $\mathbf{R}_{a}=\mathbf{r}\left(t_{a}\right)$ is the first point of $R_{\delta r}$ on the section. On the section, $R_{\delta r}$ consists of three straight segments, where circle segments connect the first and second and the second and third straight segment. Every of the five segments is parametrized from an interval $I_{i}=\left[t_{i}, \bar{t}_{i}\right] \in I_{S} i=1, . ., 5$. The length of these intervals is given by dividing the segment length by the constant parametrization speed $u_{\delta r}^{R}$. Denote the length of the intervals $I_{2}$ and $I_{4}$ by $\delta t=\pi \delta r /\left(2 u_{\delta r}^{R}\right)$ so that the polygonal limit corresponds to taking $\delta t \rightarrow 0$. The parametrization of the segments $\mathbf{r}_{i}(t)$ read

$$
\begin{aligned}
& \mathbf{r}_{1}(t)=\mathbf{R}_{a}+\left(\frac{1}{2}+u_{\delta r}^{R}\left(t-t_{a}\right)\right) \mathbf{e}_{1} \\
& \mathbf{r}_{2}(t)=\mathbf{R}_{a}+\left(1+\delta r\left(\sin \left[\frac{\pi}{2 \delta t}\left(t-t_{2}\right)\right]-1\right)\right) \mathbf{e}_{1}+\delta r\left(1-\cos \left[\frac{\pi}{2 \delta t}\left(t-t_{2}\right)\right]\right) \mathbf{e}_{2} \\
& \mathbf{r}_{3}(t)=\mathbf{R}_{a}+\mathbf{e}_{1}+\left(\delta r+u_{\delta r}^{R}\left(t-t_{2}\right)\right) \\
& \mathbf{r}_{4}(t)=\mathbf{R}_{a}+\mathbf{e}_{1}+\left(1+\delta r\left(\sin \left[\frac{\pi}{2 \delta t}\left(t-t_{4}\right)\right]-1\right)\right) \mathbf{e}_{2}+\delta r\left(1-\cos \left[\frac{\pi}{2 \delta t}\left(t-t_{4}\right)\right]\right) \mathbf{e}_{3} \\
& \mathbf{r}_{5}(t)=\mathbf{R}_{a}+\mathbf{e}_{1}+\mathbf{e}_{2}+\left(\delta r+u_{\delta r}^{R}\left(t-t_{5}\right)\right) \mathbf{e}_{3}
\end{aligned}
$$




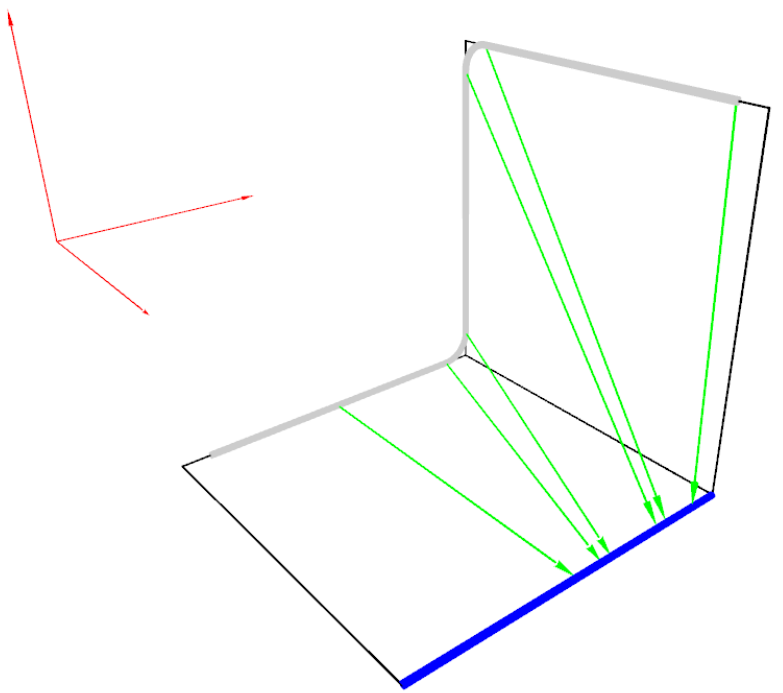

Fig. 7 A sketch of a section $\mathrm{S} \in \mathscr{G}_{\delta}$ on the configuration $C_{7}$.

We define the parameterizations $\mathbf{b}_{i}(t)$ on the intervals $I_{i}$ as

$$
\begin{gathered}
\mathbf{b}_{1}(t)=\mathbf{R}_{a}+\mathbf{e}_{3}+\left\{u_{1}^{B}\left(t-t_{1}\right)\right\} \mathbf{e}_{1} \\
\mathbf{b}_{2}(t)=\mathbf{R}_{a}+\mathbf{e}_{3}+\left\{\frac{6}{10}-\delta r+u_{2}^{B}\left(t-t_{2}\right)\right\} \mathbf{e}_{1} \\
\mathbf{b}_{3}(t)=\mathbf{R}_{a}+\mathbf{e}_{3}+\left\{\frac{6}{10}+\delta r+u_{3}^{B}\left(t-t_{3}\right)\right\} \mathbf{e}_{1} \\
\mathbf{b}_{4}(t)=\mathbf{R}_{a}+\mathbf{e}_{3}+\left\{\frac{8}{10}-\delta r+u_{4}^{B}\left(t-t_{4}\right)\right\} \mathbf{e}_{1} \\
\mathbf{b}_{5}(t)=\mathbf{R}_{a}+\mathbf{e}_{3}+\left\{\frac{8}{10}+\delta r+u_{5}^{B}\left(t-t_{5}\right)\right\} \mathbf{e}_{1}
\end{gathered}
$$

where $u_{1}^{B}=(1 / 10-\delta r) /(1 / 2-\delta r) u_{\delta r}^{R}, u_{3}^{B}=u_{5}^{B}=u_{\delta r}^{R}(2 / 10-\delta r) /(1-2 \delta r), u_{2}^{R}=u_{4}^{B}=4 \pi^{-1} \alpha u_{\delta r}^{R}$. Then, the twist can be obtained as

$$
T w(s)=\sum_{i=1}^{5} \lim _{\delta r \rightarrow 0} T w\left(B, R_{\delta r} \mid I_{i}\right)
$$


where $T w\left(B, R_{\delta r} \mid I_{i}\right)$ is the twist integral on the interval $I_{i}$. As examples, the results for $T w\left(B, R_{\delta r} \mid I_{2}\right)$ and $T w\left(B, R_{\delta r} \mid I_{3}\right)$ shall be computed. For $T w\left(B, R_{\delta r} \mid I_{2}\right)$ compute the relevant vector quantities

$$
\begin{aligned}
\mathbf{r}(t)= & \mathbf{R}_{a}+\mathbf{e}_{1}+\delta r\left(-1+\sin \left(\frac{\pi}{2 \delta t}\left(t-t_{2}\right)\right)\right) \mathbf{e}_{1} \\
& +\delta r\left(1-\cos \left(\frac{\pi}{2 \delta t}\left(t-t_{2}\right)\right)\right) \mathbf{e}_{2} \\
\mathbf{r}^{\prime}(t)= & u_{\delta r}^{R} \cos \left(\frac{\pi}{2 \delta t}\left(t-t_{2}\right)\right) \mathbf{e}_{1}+u_{\delta r}^{R} \sin \left(\frac{\pi}{2 \delta t}\left(t-t_{2}\right)\right) \mathbf{e}_{2} \\
\mathbf{r}^{\prime \prime}(t)= & -u_{\delta r}^{R} \frac{\pi}{2 \delta t} \sin \left(\frac{\pi}{2 \delta t}\left(t-t_{2}\right)\right) \mathbf{e}_{1}+u_{\delta r}^{R} \frac{\pi}{2 \delta t} \cos \left(\frac{\pi}{2 \delta t}\left(t-t_{2}\right)\right) \mathbf{e}_{2} \\
\mathbf{v}(t)= & \mathbf{e}_{3}+\left\{-\frac{4}{10}-\delta r \sin \left(\frac{\pi}{2 \delta t}\left(t-t_{2}\right)\right)+\beta_{2}\left(t-t_{2}\right)\right\} \mathbf{e}_{1} \\
& -\delta r\left(1-\cos \left(\frac{\pi}{2 \delta t}\left(t-t_{2}\right)\right)\right) \mathbf{e}_{2} \\
= & \mathbf{e}_{3}-\frac{4}{10} \mathbf{e}_{1}+O(\delta t) \\
\mathbf{v}^{\prime}(t)= & \left\{-u_{\delta r}^{R} \cos \left(\frac{\pi}{2 \delta t}\left(t-t_{2}\right)\right)+u_{2}^{B}\right\} \mathbf{e}_{1}-u_{\delta r}^{R} \sin \left(\frac{\pi}{2 \delta t}\left(t-t_{2}\right)\right) \mathbf{e}_{2}
\end{aligned}
$$

By substituting $t-t_{2} \rightarrow t$, and $\omega \equiv \pi(2 \delta t)^{-1}$ in the twist formula the integral boundaries become $[0, \delta t]$. The denominator in the integral reads

$$
\mathbf{r}^{\prime 2} \mathbf{v}^{2}-\left\langle\mathbf{r}^{\prime}, \mathbf{v}\right\rangle^{2}=u_{\delta r}^{R 2}\left(1+\left(\frac{4}{10}\right)^{2} \sin ^{2} \omega t+O(\delta t)\right)
$$

Consider, the first term in the twist formula given. It requires the triple product $\left[r^{\prime}, v^{\prime}, v\right]$, however with the three vector quantities from above one can find a constant $C$ such that $-C<\left[\mathbf{r}^{\prime}(t), \mathbf{v}^{\prime}(t), \mathbf{v}(t)\right]<C$ on the interval $[0, \delta t]$, in addition the denominator $\mathbf{r}^{\prime 2} \mathbf{v}^{2}-\left\langle\mathbf{r}^{\prime}, \mathbf{v}\right\rangle^{2}$ has no singularity on the interval, so that in the lattice limit $\delta t \rightarrow 0$ the first term (rotation term) in the twist formula vanishes. For the second term (acceleration term), it is required to compute

$$
\begin{aligned}
{\left[\mathbf{r}^{\prime}, \mathbf{r}^{\prime \prime} \mathbf{v}\right] } & =\left(u_{\delta r}^{R}\right)^{2} \frac{\pi}{2 \delta t}\left[\mathbf{e}_{1}, \mathbf{e}_{2}, \mathbf{e}_{3}\right] \\
\left\langle\mathbf{r}^{\prime}, \mathbf{v}\right\rangle & =-\frac{4}{10} u_{\delta r}^{R} \cos \omega t
\end{aligned}
$$

Thus

$$
\begin{aligned}
T w\left(B, R_{\delta r} \mid I_{2}\right) & =\frac{1}{2 \pi}\left[\mathbf{e}_{1}, \mathbf{e}_{2}, \mathbf{e}_{3}\right] \int_{0}^{\delta t} \frac{\pi}{2} \frac{d t}{\delta t} \frac{-\frac{4}{10} \cos \frac{\pi}{2 \delta t} t}{1+\left(\frac{4}{10}\right)^{2} \sin ^{2} \frac{\pi}{2 \delta t} t+O(\delta t)} \\
& =-\frac{1}{2 \pi}\left[\mathbf{e}_{1}, \mathbf{e}_{2}, \mathbf{e}_{3}\right] \int_{0}^{\frac{4}{10}}\left(1+x^{2}\right)^{-1} \\
& =-\frac{1}{2 \pi}\left[\mathbf{e}_{1}, \mathbf{e}_{2}, \mathbf{e}_{3}\right] \arctan \left(\frac{4}{10}\right)
\end{aligned}
$$

By substituting $x=\frac{4}{10} \sin \frac{\pi}{2 \delta t} t$

$$
\begin{aligned}
T w\left(B, R_{\delta r} \mid I_{2}\right) & =-\frac{1}{2 \pi}\left[\mathbf{e}_{1}, \mathbf{e}_{2}, \mathbf{e}_{3}\right] \int_{0}^{\frac{4}{10}}\left(1+x^{2}\right)^{-1} \\
& =-\frac{1}{2 \pi}\left[\mathbf{e}_{1}, \mathbf{e}_{2}, \mathbf{e}_{3}\right] \arctan \left(\frac{4}{10}\right)
\end{aligned}
$$


On the third interval $I_{3}$

$$
\begin{aligned}
\mathbf{r}(t) & =\mathbf{R}_{a}+\mathbf{e}_{1}+\left(\delta r+u_{\delta r}^{R}\left(t-t_{k+1}^{+}\right)\right) \mathbf{e}_{2} \\
\mathbf{r}^{\prime}(t) & =u_{\delta r}^{R} \mathbf{e}_{2} \\
\mathbf{r}^{\prime \prime}(t) & =0 \\
\mathbf{v}(t) & =\mathbf{e}_{3}+\left\{-\frac{4}{10}+\delta r+u_{3}^{B}\left(t-t_{3}\right)\right\} \mathbf{e}_{1}-\left(\delta r+u_{\delta r}^{R}\left(t-t_{3}\right)\right) \mathbf{e}_{2} \\
& =\mathbf{e}_{3}+\left\{u_{3}^{B}\left(t-t_{3}\right)-\frac{4}{10}\right\} \mathbf{e}_{1}-u_{\delta r}^{R}\left(t-t_{3}\right) \mathbf{e}_{2}+O(\delta t) \\
\mathbf{v}^{\prime}(t) & =u_{3}^{B} \mathbf{e}_{1}-u_{\delta r}^{R} \mathbf{e}_{2}
\end{aligned}
$$

Substitute $t-t_{3} \rightarrow t$ in the integral, then $t \in\left[0,\left(u_{\delta r}^{R}\right)^{-1}(1-2 \delta r)\right]$ and the denominator

$$
\mathbf{r}^{\prime 2} \mathbf{v}^{2}-\langle\mathbf{r}, \mathbf{v}\rangle^{2}=\left(u_{\delta r}^{R}\right)^{2}\left\{1+\left\{u_{3}^{B} t+\delta r-\frac{4}{10}\right\}^{2}\right\}
$$

has no singularity on the integration interval. Because $r^{\prime \prime}=0$, the acceleration term vanishes automatically and only the rotation term remains.

$$
\left[\mathbf{v}^{\prime}, \mathbf{r}^{\prime}, \mathbf{v}\right]=\left[\mathbf{e}_{1}, \mathbf{e}_{2}, \mathbf{e}_{3}\right] u_{\delta r}^{R} u_{3}^{B}
$$

Therefore, after substituting $x=u_{3}^{B} t+\delta r-\frac{4}{10}$

$$
\begin{aligned}
T w\left(B, R_{\delta r} \mid I_{3}\right)= & \frac{1}{2 \pi}\left[\mathbf{e}_{1}, \mathbf{e}_{2}, \mathbf{e}_{3}\right] \int_{\delta r-4 / 10}^{u_{3}^{B}(1-2 \delta r)+\delta r-4 / 10} \frac{1}{1+x^{2}}+O(\delta t) \\
= & \frac{1}{2 \pi}\left[\mathbf{e}_{1}, \mathbf{e}_{2}, \mathbf{e}_{3}\right]\left\{\arctan \left(\frac{u_{3}^{B}}{u_{\delta r}^{R}}(1-2 \delta r)+\delta r-4 / 10\right)\right. \\
& -\arctan (\delta r-4 / 10)\}+O(\delta t)
\end{aligned}
$$

In the polygonal limit $\left(u_{\delta r}^{R}\right)^{-1} u_{3}^{B}=2 / 10$ so that

$$
\lim _{\delta t \rightarrow 0} T w\left(B, R_{\delta r} \mid I_{3}\right)=\frac{1}{2 \pi}\left[\mathbf{e}_{1}, \mathbf{e}_{2}, \mathbf{e}_{3}\right]\{\arctan (4 / 10)-\arctan (2 / 10)\}
$$

The integrals on $I_{4}$ and $I_{5}$ can be treated analogously. Compute $\lim _{\delta t \rightarrow 0} T w\left(B, R_{\delta r} \mid I_{4}\right)=0$ and $\lim _{\delta t \rightarrow 0} T w\left(B, R_{\delta r} \mid I_{5}\right)=\frac{1}{2 \pi}\left[e_{1}, e_{2}, e_{3}\right] \arctan (2 / 10)$. Therefore, the twist of the sequence reads

$$
\begin{aligned}
T w(c) & =\sum_{i=1}^{4} \lim _{\delta t \rightarrow 0} T w\left(I_{i}\right) \\
& =0
\end{aligned}
$$




\section{University Library}

\section{- M M N E R VA A gateway to Melbourne's research publications}

Minerva Access is the Institutional Repository of The University of Melbourne

Author/s:

Dagrosa, E;Owczarek, AL

Title:

Generalizing Ribbons and the Twist of the Lattice Ribbon

Date:

2014-04-01

Citation:

Dagrosa, E. \& Owczarek, A. L. (2014). Generalizing Ribbons and the Twist of the Lattice

Ribbon. JOURNAL OF STATISTICAL PHYSICS, 155 (2), pp.392-417. https://doi.org/10.1007/ s10955-014-0941-y.

Persistent Link:

http://hdl.handle.net/11343/283022 